May, 1995

\title{
Topological Landau-Ginzburg theory with a rational potential and the dispersionless KP hierarchy
}

\author{
Shogo Aoyama ${ }^{1}$ and Yuji Kodama ${ }^{2} *$ \\ ${ }^{1}$ Department of Physics, Shizuoka University \\ Shizuoka 422, Japan \\ E-mail: spsaoya@ipcs.shizuoka.ac.jp \\ ${ }^{2}$ Department of Mathematics, Ohio State University \\ Columbus, $O H$ 43210, USA \\ E-mail:kodama@math.ohio-state.edu
}

\begin{abstract}
Based on the dispersionless KP (dKP) theory, we give a comprehensive study of the topological Landau-Ginzburg (LG) theory characterized by a rational potential. Writing the dKP hierarchy in a general form, we find that the hierarchy naturally includes the dispersionless (continuous) limit of Toda hierarchy and its generalizations having finite number of primaries. Several flat solutions of the topological LG theory are obtained in this formulation, and are identified with those discussed by Dubrovin. We explicitly construct gravitational descendants for all the primary fields. Giving a residue formula for the 3-point functions of the fields, we show that these 3-point functions satisfy the topological recursion relation. The string equation is obtained as the generalized hodograph solutions of the dKP hierarchy, which show that all the gravitational effects to the constitutive equations (2-point functions) can be renormalized into the coupling constants in the small phase space.
\end{abstract}

\footnotetext{
* Supported in part by NSF grant DMS-9403597
} 


\section{$\S 1 . \quad$ Introduction}

The equivalence of the multi-matrix model to the 2-d gravity coupled with comformal matter [KPZ, DK, Da] was shown by solving the generalized $\mathrm{KdV}$ hierarchy [BKa, DS, GM, BDKS, CGM, Do, BDSS]. Soon later the 2-d topological field theory (TFT) coupled to topological gravity was constructed, which encodes the essential features of these three disciplines, i.e., local coordinate independence, scaling property and integrability [W]. It was then shown that the multi-matrix model is equivalent to the topological conformal matter system coupled to the topological gravity [DW, DVV2]. The TFT coupled to the gravity can be studied in a purely topological approach based on the cohomology of the physical observables - This cohomological nature is inherent also in the minimal conformal matter system and the generalized $\mathrm{KdV}$ hierarchy, i.e. fusion rules and polynomial ring (in the dispersionless limit) respectively.

In this regard the Landau-Ginzburg (LG) theory, which had already been useful in the classification of the conformal field theory [VW], also came close to the arena of these subjects. Namely, through the correspondence of the LG potential to the Lax operator of the generalized KdV hierarchy, it gave a most convenient picture for understanding the ring structure of the hierarchy (in the dispersionless limit), i.e. a deformation of the ring by the gravitational couplings. In fact, genuszero correlation functions of an arbitrary TFT coupled to 2-d topological gravity are determined by an appropriate LG potential (LG picuture).

Among these theories the TFT coupled to the gravity in [W] is most general, dealing not only with non-conformal matter, but also the geometry of the matter system. Of course, the KdV hierarchy can be further generalized to the $\mathrm{KP}$ hierarchy with a certain reduction, but it is not clear how the generalized theory comes across the latter subject.

A coupled system of the gravity and topological matter fields $\phi^{\alpha}$ (primaries) is given by the action

$$
S=S_{0}+\sum_{\substack{\alpha \in \text { primaries } \\ N \geq 0}} t_{N, \alpha} \int_{\Sigma} \sigma_{N}\left(\phi^{\alpha}\right),
$$

with $S_{0}$ the basic action obtained by twisting an ordinary model, $\sigma_{N}\left(\phi^{\alpha}\right)$ the $N$ th 
gravitational desecndant of $\phi^{\alpha}$, and $t_{N, \alpha}$ the coupling constants. It is essentially characterized by genus-zero 3-point functions $\left\langle\phi^{\alpha} \phi^{\beta} \phi^{\gamma}\right\rangle$. The TFT is then defined such that, when the descendants couplings are turned off at certain values, the 3-point functions $\left\langle\phi^{\alpha} \phi^{\beta} \phi^{\gamma}>:=c^{\alpha \beta \gamma}\right.$ of the theory satisfy a set of equations,

$$
\begin{aligned}
\eta^{\alpha \beta} & :=c^{\alpha \beta 0}=\text { constant, } \quad \text { (flatness of metric) } \\
\frac{\partial}{\partial t_{\delta}} c^{\alpha \beta \gamma} & =\frac{\partial}{\partial t_{\alpha}} c^{\delta \beta \gamma}, \quad \text { (integrability) } \\
\sum_{\lambda, \mu=\text { primaries }} c^{\alpha \beta \lambda} \eta_{\lambda \mu} c^{\mu \gamma \delta} & =\sum_{\lambda, \mu=\text { primaries }} c^{\alpha \gamma \lambda} \eta_{\lambda \mu} c^{\mu \beta \delta} \quad \text { (associativity) }
\end{aligned}
$$

Here $t_{\alpha}:=t_{0, \alpha}$ are the primary couplings in (1.1), and $\eta_{\lambda \mu}$ is the inverse of $\eta^{\lambda \mu}$. The set of these equations (1.2) is called the WDVV equation [Du1], and the solutions of this equation describe the topological limits of the TFT coupled to the gravity (the "flat" solutions). In this paper, we call the space of primary couplings $t_{\alpha}$ alone the "small" phase space, and the entire coupling space the "large" phase space.

The genus-zero limit of certain TFT's coupled to the gravity is equivalent to the dispersionless limit of the KP (dKP) hierarchy with a reduction. In [DVV1] the flat solutions to the A-, D-, and E- (ADE-) models of the topological minimal models were obtained by the LG approach. In particular, a structure of the dKP hierarchy was found for the case of the A-model, where the fusion ring of the primaries is given by a "polynomial" ring with one variable $p$, the quasi-momentum, over an ideal given by the LG potential. The approach based on the dKP hierarchy was further extended in [AK, Kr2, T] by introducing a "rational" LG potential, which includes the D-model and its extention [T]. TFT's with a rational potential appear in several literatures, such as a classical limit of the multi-matrix model $[\mathrm{BX}]$, the multi-field representations of the KP hierarchy [ANPV], and the symmetry constrained KP hierarchy [OS]. (They are all equivalent, and have the same Lax operators.)

A classification of the flat solutions of the WDVV equation was given in [Du2], by studying the Frobenius manifold, but not in the dKP approach. The 
group theoretical structure behind the solution was understood by associating the scale dimensions of the primaries with the degrees of the Coxeter group. It was then shown that all the classified solutions fall into the ADE-series or the relatives by some truncations $[\mathrm{Z}]$. Quite recently the solution of the $C P^{1}$ model [W, DW] was also found in the LG approach [EY]. Of course, there are other flat solutions $[\mathrm{W}, \mathrm{DW}]$ for which the LG description is not yet known.

All these flat solutions are obtained as the topological limits of the TFT coupled to the gravity. The generalizations of the analysis to the large phase space has been carried out in a rather limited extent, except in [AK, Kr2, LP]. The framework based on the cohomology in [W, DW] provides a perturbative method to study the TFT in the large phase space. But the integrable structure behind such a solution of the theory remains obscure. In this regard, the approach based on the dKP hierarchy with the LG picture prevails that by the cohomological approach.

In this paper we give a comprehensive study of the topological LG theory having a rational potential based on the dKP hierarchy previously proposed in [AK]. The paper is organized as follows: In Section 2, we give a mathematical background of the dKP theory. The main purpose in this section is to rewrite the dKP hierarchy in a general form, and show that it includes several fusion rings for a single rational potential. In particular, the dispersionless limit of Toda (dToda) hierarchy [TT1, Ko1, SV] is shown to be naturally included in this formulation.

Section 3 introduces the universal coordinates for the deformation variables of the rational potential. Then writing the dKP hierarchy with these coordinates, we show the integrability of the hierarchy. The generalized Gel'fand-Dikey potentials in the hierarchy are also given explicitly in a residue formula. We also find several flat solutions corresponding to the topological limits of our TFT model.

In Section 4, based on the integrability of the hierarchy, we define the free energy and the N-point functions of the TFT. The 3-point function is explicitly expressed by a residue formula. We also define another type of the free energy and the corresponding $\mathrm{N}$-point functions, which are the functions of the universal coordinates. But the 2-point functions of both types are found to be a unique object. Namely these 2-point functions calculated from the respective free energies 
coincide. Moreover, the 2-point functions are free from the choice of the primary rings obtained in the general formulation of the dKP hierarchy. This leads to a symmetry of the WDVV equation discussed in [Du2]. A hamiltonian form for the dKP hierarchy is also found in terms of 1-point functions which are also given explicitly.

Section 5 defines the gravitational descendants for all the primary fields. In particular, we elaborate the descendants of the primary $\phi^{-1}$ which is a typical flow in the dToda hierarchy. The descendant fields of $\phi^{-1}$ for the dToda hierarchy $\left(\mathrm{CP}^{1}\right.$-model $)$ were first found in $[\mathrm{EY}]$, but in a rather heuristic way. Here we give a mathematical justification to their result. Then we show that the topological recursion relation for these descendants naturally inheres in the framework of the dKP theory.

In Section 6, we show that the solution of the dKP hierarchy is completely determined in the small phase space. This implies that all the gravitational effects to the constitutive equations (2-point functions) can be renormalized in the primary couplings. This is precisely the theorem obtained in $[\mathrm{KG}]$, and the string equation is then obtained as a consequence of this theorem. The solution of the string equation is given algebraically in the generalized hodograph transform, and can be explicitly obtained as a perturbative solution with small gravitational couplings. This is the well-known procedure of the renormalization in the quantum field theory.

In Section 7, we discuss the critical phenomena based on our LG theory. The main objective here is to study the scaling behavior of the solution of the dKP hierarchy in the small phase space. The critical exponents of the free energy and the primaries are calculated for the scaling models, which has no scaling violation term, such as log-solution. Among the LG theories having different type of rational potentials, we find that there exists an equivalent pair in the sense that two theories in this pair give the same scaling behavior at all the critical points. We also discuss a phase transition corresponding to a singularity (shock formation) appearing in the string equation. The singularity may be regularized by adding a finite genus effect to the dKP hierarchy (the Whitham approach) [BKo, Du1, Kr2].

In Section 8, we illustrate the results obtained in this paper by taking several 
explicit examples including the $\mathrm{CP}^{1}$-model [DW, EY], and especially we give the corresponding free energies for the flat solutions. We also provide a detail analysis of the terms including log-singularity in Appendix A, and a brief overview of the dKP theory in Appendix B.

In this paper, we restrict ourself to the analysis of the TFT in the genuszero limit, which corresponds to a spherical approximation of the world sheet in the string theory. Effects of finite genus to the world sheet may be studied by replacing the quasi-momentum $p$ in the rational potential by a differential symbol $\partial$ (i.e. the multi-matrix models of $[\mathrm{BX}, \mathrm{Da}]$ ), or by promoting the potential into a matrix form $[\mathrm{KO}]$. Quantization of the dKP theory may be also obtained by the Moyal deformation [S]. We will study these in a future communication.

\section{$\S 2$. Preliminary on the dispersionless KP hierarchy}

In this paper we study a topological Landau-Ginzburg (LG) theory with a rational potential given by a Laurent polynomial of $p$ and $(p-s)^{-1}$ [AK, Kr2],

$$
\begin{aligned}
W= & W(p ; v, s) \\
= & \frac{1}{n+1} p^{n+1}+v_{n-1} p^{n-1}+\cdots \cdots+v_{0} \\
& \quad+\frac{v_{-1}}{p-s}+\cdots \cdots+\frac{v_{-(m-1)}}{(m-1)(p-s)^{m-1}}+\frac{v_{-m}}{m(p-s)^{m}} .
\end{aligned}
$$

Here the variable $p$ represents the quasi-momentum of the field, and the (complex) coefficients $v_{a},-m \leq a \leq n-1$ and $s$ are the deformation variables of the potential. At the singularities $p=\infty$ and $p=s$ of the $\mathrm{W}$ potential we introduce the local coordinates $\lambda$ and $\mu$,

$$
\begin{aligned}
& \lambda=p+O\left(\frac{1}{p}\right), \quad \text { for large } p, \\
& \mu=\frac{\sqrt[m]{v_{-m}}}{p-s}+O(1), \quad \text { for small } p-s,
\end{aligned}
$$

which are also globally defined through $[\mathrm{AK}, \mathrm{T}]$

$$
W=\frac{\lambda^{n+1}}{n+1}=\frac{\mu^{m}}{m}
$$


The main objective in this paper is to study deformation of the $\mathrm{W}$ potential in the framework of the LG theory for TFT, where the deformation is induced by coupling constants. Here we introduce an infinite number of coupling constants $t_{i}$ for $i \in \mathbf{Z}$, and consider the deformation variables $\left\{v_{a}\right\}$ and $s$ to be functions of $t_{i}$ 's. Then we assume the $\mathrm{W}$ potential to satisfy the following flow equations of the deformation,

$$
\frac{\partial W}{\partial t_{i}}=\left\{Q^{i}, W\right\}:=\frac{\partial Q^{i}}{\partial p} \frac{\partial W}{\partial t_{0}}-\frac{\partial Q^{i}}{\partial t_{0}} \frac{\partial W}{\partial p}, \quad \text { for } \quad i \in \mathbf{Z}
$$

with the genrators $Q^{i}$ defined by

$$
Q^{i}:=\left\{\begin{array}{lrl}
{\left[\frac{1}{i+1} \lambda^{i+1}\right]_{+},} & \text {for } \quad 0 \leq i<\infty, \\
{[\log \lambda]_{+}-[\log \mu]_{-},} & \text {for } i=-1, \\
-\left[\frac{1}{|i|-1} \mu^{|i|-1}\right]_{-}, & \text {for } \quad-\infty<i \leq-2 .
\end{array}\right.
$$

The symbols $[\cdot]_{+}$and $[\cdot]_{-}$indicate the parts of non-negative powers in $p$ and negative powers in $p-s$, respectively. In Appendix A, we give a precise definition of these \pm projections, and also provide explicit caluculations of the terms including the $\log$ - terms, $\log \lambda$ and $\log \mu$. In particular, one can show that $Q^{-1}=\log (p-s)$, which was previously used in $[\mathrm{AK}, \mathrm{Kr} 2]$. It should be also noted that the definition of $Q^{-1}$ in (2.5) is natural, even though it looks complicated. Indeed, this definition clearly shows that $Q^{-1}$ is a generator of degree zero in $p$ having contributions from both singularities $p=\infty$ and $p=s$ in the rational potential $W$.

Eq. (2.4) is nothing but the dispersionless $\mathrm{KP}$ (dKP) hierarchy [KG, TT2, $\mathrm{Kr} 1$, Du1] with the reduction given by the $\mathrm{W}$ potential (2.1). We refer the set of eqs.(2.4) the dKP hierarchy in this paper, even though we mainly concern with the reduced one. In Appendix B, we briefly summarize the theory of the dKP hierarchy. Note here that each deformation in (2.4) should be independent from the others. Namely we have to have the compatibility conditions among the flows in (2.4). In order to confirm the conditions, we fisrt note:

Lemma 2.1. The generators $Q^{i}$ satisfy the zero curvature condition,

$$
\frac{\partial Q^{i}}{\partial t_{j}}-\frac{\partial Q^{j}}{\partial t_{i}}+\left\{Q^{i}, Q^{j}\right\}=0, \quad \text { for } \quad i, j \in \mathbf{Z}
$$


Proof. Here we give a proof in the general form: Let $F$ and $G$ be the functions of either $\lambda$ or $\mu$, and define $F_{ \pm}:=[F]_{ \pm}$and $G_{ \pm}:=[G]_{ \pm}$. The flow parameters corresponding to $F_{ \pm}$and $G_{ \pm}$are denoted by $t_{ \pm}$and $s_{ \pm}$, respectively. Then, for the case with $F_{+}$and $G_{-}$, we have

$$
\begin{aligned}
\frac{\partial F_{+}}{\partial s_{-}} & =\left[\frac{\partial F}{\partial s_{-}}\right]_{+}=\left\{G_{-}, F\right\}_{+} \\
& =\left\{G_{-}, F_{+}\right\}-\left\{G_{-}, F_{+}\right\}_{-} \\
& =\left\{G_{-}, F_{+}\right\}+\frac{\partial G_{-}}{\partial t_{+}} .
\end{aligned}
$$

For the case with $F_{+}$and $G_{+}$, that is, both $F$ and $G$ are the functions of $\lambda$, we have

$$
\begin{aligned}
\frac{\partial F_{+}}{\partial s_{+}} & =\left[\frac{\partial F}{\partial s_{+}}\right]_{+}=\left\{G_{+}, F\right\}_{+} \\
& =\left\{G_{+}, F_{+}\right\}+\left\{G_{+}, F_{-}\right\}_{+} \\
& =\left\{G_{+}, F_{+}\right\}+\left\{G, F_{-}\right\}_{+} \\
& =\left\{G_{+}, F_{+}\right\}-\left\{G, F_{+}\right\}_{+} \\
& =\left\{G_{+}, F_{+}\right\}+\frac{\partial G_{+}}{\partial t_{+}} .
\end{aligned}
$$

Using these results, the case including $Q^{-1}$ can be also shown by a similar but careful computation (see Appendix A for a detail).

We then obtain:

Proposition 2.2. The flows in eq. (2.4) are compatible (or commuting) for all $t_{i}{ }^{\prime} s$,

$$
\frac{\partial}{\partial t_{i}} \frac{\partial}{\partial t_{j}} W=\frac{\partial}{\partial t_{j}} \frac{\partial}{\partial t_{i}} W
$$

Proof. $\quad$ From eq. (2.4) the compatibility condition can be written in the form

$$
\frac{\partial}{\partial t_{i}} \frac{\partial}{\partial t_{j}} W-\frac{\partial}{\partial t_{j}} \frac{\partial}{\partial t_{i}} W=\left\{\frac{\partial Q^{j}}{\partial t_{i}}-\frac{\partial Q^{i}}{\partial t_{j}}+\left\{Q^{j}, Q^{i}\right\}, W\right\} .
$$

Use of Lemma 2.1 completes the proof.

We thus see that the flows commute if those generators are the functions of $\lambda$ or/and $\mu$ only. Proposition 2.2 implies that the dKP hierarchy (2.4) possesses an infinite number of symmetries inducing the conservation laws (see Theorem.3.4), 
and leading to its integrability. This gives the main reason why we use the dKP hierarchy to express the deformation with an infinite number of coupling constants.

Let us now consider a ring of Laurent polynomials over $\mathbf{C}$, denoted by $\mathbf{C}\left[p,(p-s)^{-1}\right]$. A basis for this ring may be given by

$$
\phi^{i}:=\frac{d Q^{i}}{d p}, \quad i \in \mathbf{Z} .
$$

Namely $\phi^{i}$ is a Laurent polynomial of degree $i \in \mathbf{Z}$. Introducing an ideal given by

$$
\frac{d W}{d p}:=W^{\prime}=\phi^{n}-\phi^{-(m+1)}=0,
$$

we have a (commutative and associative) finite dimensional rational ring of dimension $n+m+1$,

$$
\mathcal{R}:=\frac{\mathbf{C}\left[p,(p-s)^{-1}\right]}{W^{\prime}(p)} .
$$

A basis of this ring $\mathcal{R}$ can be taken to be a set of $n+m+1$ consecutive elements in eq. (2.8), including $\phi^{0}$ as an identity element of the ring. We thus consider here a topological field theory with $n+m+1$ primary fields. Since $\phi^{n}$ and $\phi^{-(m+1)}$ are identified by eq. (2.9), a natural basis may be chosen as

$$
\left\{\phi^{\alpha}: \alpha \in \Delta_{n, m}\right\},
$$

where the set of indicies $\Delta_{n, m} \subset \mathbf{Z}$ is given by

$$
\Delta_{n, m}:=\{i \in \mathbf{Z}:-m \leq i \leq n\} .
$$

The fields $\phi^{\alpha}$ in (2.11) are called the "primaries", which describe the matter fields of our TFT, while the other $\phi$ 's the "gravitational descendants". (Throughout this paper, we use the Greek letters for the primary indices, and the Roman letters for all indices including both primary and descendants indices.) The TFT is then described by an action,

$$
\begin{aligned}
S & =S_{0}+\sum_{i \in \mathbf{Z}} t_{i} \phi^{i} \\
& =S_{0}+\sum_{\alpha \in \Delta_{n, m}} t_{\alpha} \phi^{\alpha}+\sum_{i \in \mathbf{Z} \backslash \Delta_{n, m}} t_{i} \phi^{i},
\end{aligned}
$$


where $S_{0}$ is the matter sector action. In this formula, the coupling constants $t_{\alpha}$ with $\alpha \in \Delta_{n, m}$ are called the primary couplings describing the deformation of the matter sector of the TFT, while all the others the gravitational couplings describing the gravitational deformations.

In the view point of the dKP hierarchy (2.4), the fields $\phi^{i}$ and the coupling constants $t_{i}$ are related by

$$
\frac{\partial W\left(p_{*}\right)}{\partial t_{i}}=\phi^{i}\left(p_{*}\right) \frac{\partial W\left(p_{*}\right)}{\partial t_{0}},
$$

where $p_{*}$ is a root of the ideal $W^{\prime}(p)=0$, denoted by $p_{*} \in \operatorname{Ker} W^{\prime}$. This is the Riemann invariant form of the flow equation (2.4) which is a quasi-linear system of first order equations for the deformation variables $v_{a}$ and $s$ in $W$. In this form of the dKP hierarchy, we note that the primary coupling $t_{0}$ plays a particular role, called the "cosmological constant" in the string theory, and the corresponding field is the identity $\phi^{0}$. These are customarily denoted as $t_{0}=t_{\mathcal{P}}$ and $\phi^{0}=\mathcal{P}$, where $\mathcal{P}$ is called the "puncture" operator. However, writing the dKP hierarchy (2.4) in a more general form, one can define a different set of primary fields, and associate $t_{\mathcal{P}}$ with an other primary coupling $t_{\alpha_{0}}$ for some $\alpha_{0} \neq 0$, whose field is of course the identity of this set. This general form of the dKP hierarchy may be given by

$$
\left\{Q_{j}, W\right\}_{i}=\left\{Q_{i}, W\right\}_{j}
$$

where $\{A, B\}_{i}$ represents the Poisson bracket with $\left(p, t_{i}\right)$ pair, i.e.

$$
\{A, B\}_{i}:=\frac{\partial A}{\partial p} \frac{\partial B}{\partial t_{i}}-\frac{\partial A}{\partial t_{i}} \frac{\partial B}{\partial p} .
$$

This is derived from (2.4) and (2.6), and the dKP hierarchy in (2.4) corresponds to the case with $j=0$. From the form (2.15) with fixed $j=\alpha_{0} \in \Delta_{n, m}$, the flow equation for the $\mathrm{W}$ potential similar to (2.14) takes the form,

$$
\frac{\partial W\left(p_{*}\right)}{\partial t_{i}}=\tilde{\phi}^{i}\left(p_{*}\right) \frac{\partial W\left(p_{*}\right)}{\partial t_{\alpha_{0}}}:=\frac{\phi^{i}\left(p_{*}\right)}{\phi^{\alpha_{0}}\left(p_{*}\right)} \frac{\partial W\left(p_{*}\right)}{\partial t_{\alpha_{0}}} .
$$

This defines a set of new primary fields with a fixed $\alpha_{0} \in \Delta_{n, m}$,

$$
\left\{\tilde{\phi}^{\alpha}:=\phi^{\alpha} / \phi^{\alpha_{0}} \quad\left(\bmod W^{\prime}\right): \alpha \in \Delta_{n, m}\right\},
$$


whose identity element is given by $\tilde{\phi}^{\alpha_{0}}$. This implies that we have $n+m+1$ different choices of the puncture operator, $\mathcal{P}=\tilde{\phi}^{\alpha_{0}}$, and the cosmological constant $t_{\mathcal{P}}=t_{\alpha_{0}}$. Note that each new field $\tilde{\phi}^{\alpha}$ is also defined as an element of the ring $\mathcal{R}$ in $(2.10)$. One should also note that the hierarchy (2.15) is mathematically equivalent to (2.4), and the solutions of this hierarchy are of course the same as those of (2.4). However, as we explained above, the physical significance of the flow parameters $t_{\alpha}$ is different, and this observation will be useful to construct various solutions relevant to our TFT. In particular, the dKP hierarchy in (2.15) with $j=\alpha_{0}=-1$ turns out to be the dispersionless (or continuous) limit of the generalized Toda hierarchy [Ko1], and introducing $P:=p-s,(2.15)$ becomes

$$
\frac{\partial W}{\partial t_{i}}=P\left(\frac{\partial Q_{i}}{\partial P} \frac{\partial W}{\partial t_{-1}}-\frac{\partial Q_{i}}{\partial t_{-1}} \frac{\partial W}{\partial P}\right)
$$

The right hand side gives the Poisson bracket for the dispersionless Toda hierarchy [TT1]. The basis of this ring is then given by

$$
\left\{\tilde{\phi}^{\alpha}=P \phi^{\alpha}\left(\bmod W^{\prime}\right): \alpha \in \Delta_{n, m}\right\} \subset \mathbf{C}\left[P, P^{-1}\right]
$$

and the $\mathrm{W}$ potential is

$$
W=\frac{1}{n+1} P^{n+1}+w_{n} P^{n}+\cdots+w_{0}+\frac{w_{-1}}{P}+\frac{w_{-2}}{2 P^{2}}+\cdots+\frac{w_{-m}}{m P^{m}} .
$$

With the ideal (2.9), the fusion algebra on the $\operatorname{ring} \mathcal{R}$ is defined by the product rule,

$$
\phi^{\alpha} \phi^{\beta}=\sum_{\gamma \in \Delta_{n, m}} c_{\gamma}^{\alpha \beta} \phi^{\gamma} \quad\left(\bmod W^{\prime}\right), \quad \text { for } \quad \alpha, \beta \in \Delta_{n, m}
$$

with the structure constants $c_{\gamma}^{\alpha \beta}$. Associativity of the fusion algebra plays a fundamental role for the TFT described by the $\mathrm{W}$ potential. It is then an important subject to study the structure constants as functions of the coupling constants $t_{i}$ for $i \in \mathbf{Z}$. We will study the fusion algebra (2.21) in terms of the 3-point functions in Section 4.

As a final remark of this section, we note that our choice of the rational ring on $\mathbf{C}\left[p,(p-s)^{-1}\right]$ can be naturally extended to a more general case with multipoles at $p=s_{i}, i=1, \cdots, l$, proposed in [ANPV, BX, Kr2]. In fact, because of 
asymmetric form of $p$ and $(p-s)^{-1}$, it is immediate to see that a ring in this general case is defined on the Laurent polynomials in $\mathbf{C}\left[p,\left(p-s_{1}\right)^{-1}, \cdots,\left(p-s_{l}\right)^{-1}\right]$.

\section{$\S 3$. The dKP hierarchy in the universal coordinates}

The dKP hierarchy (2.4) defines the flows of the variables $v_{a},-m \leq a \leq$ $n-1$ and $s$. In this section we reformulate the dKP hierarchy (2.4) in terms of new variables, that is, a reparametrization of the deformation variables. These new variables which we refer as the "universal coordinates" are introduced as follows: First we invert eqs. (2.2a) and (2.2b) in terms of $p$ respectively (see also Appendix B for a motivation of this procedure),

$$
\begin{aligned}
& p=\lambda-\frac{u^{0}}{\lambda}-\cdots \cdots \cdots-\frac{u^{i}}{\lambda^{i+1}}-\cdots, \quad \text { for large } \lambda \\
& p=u^{-1}+\frac{u^{-2}}{\mu}+\cdots \cdots+\frac{u^{-i}}{\mu^{i-1}}+\cdots, \quad \text { for large } \mu .
\end{aligned}
$$

Then we have:

Lemma 3.1. The coefficients $u^{i}$ for $i \in \mathbf{Z}$ can be expressed as the residue formulae,

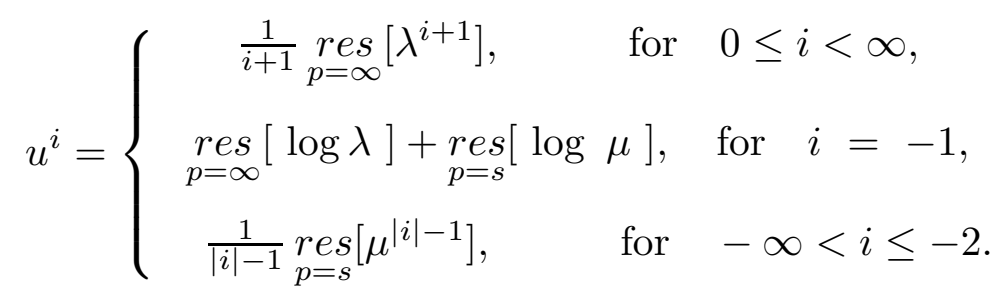

(The residue formula is defined in the usual way as (A.3) in Appendix A.)

Proof. These formulae except $i=-1$ can be shown by replacing the differential $d p$ in the residue integral with that of the local coordinate, i.e. $d p=(d p / d \lambda) d \lambda$ or $d p=(d p / d \mu) d \mu$. As explained in Appendix A, the $u^{-1}$ is evaluated as

$$
\begin{aligned}
& u^{-1}=\underset{p=\infty}{\operatorname{res}}[\log \lambda]+\underset{p=s}{\operatorname{res}}[\log \mu]=\underset{p=\infty}{\operatorname{res}}[\log p]-\underset{p=s}{\operatorname{res}}[\log (p-s)] \\
& =\frac{1}{2 \pi i}\left[\oint_{C_{\infty}} \log p d p-\oint_{\tilde{C}_{s}} \log (p-s) d p\right]=\frac{1}{2 \pi i} \oint_{C_{\infty}} \log \left(\frac{p}{p-s}\right) d p=s,
\end{aligned}
$$


where the contour $C_{\infty}$ is taken arround $p=\infty$, and $\tilde{C}_{s}$ to surround a branch cut between $p=s$ and $p=\infty$, in both the directions of counter-clockwise.

Note in (3.2) that the coefficients $u^{\alpha}$ for $\alpha \in \Delta_{n, m}$ are determined from the deformation variables $v_{a}$ and $\mathrm{s}$ in eq. (2.1), while the others are polynomials of these $u^{\alpha}$ (see Proposition 3.3 for their explicit forms). These variables $u^{\alpha}$ play an important role throughout this paper, and we call them the universal coordinates of the deformation. One of the main purpose of this paper is to construct them as functions of the coupling constants $t^{i}$ by solving the dKP hierarchy (2.4).

We note that the universal coordinates $u^{\alpha}$ are related to the primaries $\phi^{\alpha}$ through the $\mathrm{W}$ potential:

Proposition 3.2. For each primary index $\alpha \in \Delta_{n, m}$, we have

$$
\frac{\partial W}{\partial u^{\alpha}}=\phi_{\alpha}:=\sum_{\beta \in \Delta_{n, m}} \eta_{\alpha \beta} \phi^{\beta}, \quad\left(\bmod W^{\prime}\right)
$$

where a metric $\eta_{\alpha \beta}$ is defined by

$$
\eta_{\alpha \beta}=\left\{\begin{array}{cl}
\delta_{\alpha+\beta, n-1}, & \text { for }-1 \leq \alpha, \beta \leq n \\
\delta_{\alpha+\beta,-m-2}, & \text { for }-m \leq \alpha, \beta \leq-2, \\
0, & \text { otherwise }
\end{array}\right.
$$

(Throughout this paper, we use $\eta_{\alpha \beta}=\eta^{\alpha \beta}$ for lowering and raising the primary indices.)

Proof. We differentiate $u^{\alpha}$ in (3.2) with respect to $u^{\beta}$ to find for $0 \leq \alpha, \beta \leq n$,

$$
\delta_{\alpha, \beta}=\underset{p=\infty}{\operatorname{res}}\left[\lambda^{\alpha} \frac{\partial \lambda}{\partial u^{\beta}}\right]=\underset{p=\infty}{\operatorname{res}}\left[\lambda^{\alpha-n} \frac{\partial W}{\partial u^{\beta}}\right] .
$$

On the other hand the definition of $\phi^{\beta}$ in (2.8) leads to

$$
\underset{p=\infty}{\operatorname{res}}\left[\lambda^{\alpha-n} \phi^{\beta}\right]=\underset{\lambda=\infty}{\operatorname{res}}\left[\lambda^{\alpha-n} \lambda^{\beta}\right]=\delta_{\alpha+\beta, n-1}
$$

for $0 \leq \beta \leq n$. By inspecting (3.1) we note for $0 \leq \beta \leq n$

$$
\frac{\partial W}{\partial u^{\beta}} \in \mathbf{C}[p]
$$


Then the uniqueness of polynomials which are orthogonal to $\lambda^{\alpha-n}$ gives eq

for $0 \leq \alpha \leq n$. A similar calculation leads to the cases for $-m \leq \alpha<0$.

The formula (3.3) is a Legendre transform between

$$
\left\{u^{\alpha}: \alpha \in \Delta_{n, m}\right\} \quad \text { and } \quad\left\{\phi^{\alpha}: \alpha \in \Delta_{n, m}\right\}
$$

with the generator $W$, i.e. $d W=\sum_{\alpha \in \Delta_{n, m}} \phi^{\alpha} d u_{\alpha}$, and gives an inversion formula for a reconstruction of the $\mathrm{W}$ potential from the universal coordinates $u^{\alpha}$. In fact we have:

Proposition 3.3. The variables $v_{a},-m \leq a \leq n-1$ and $s$ can be found as the functions of the universal coordinates $u_{\alpha}:=\sum_{\beta \in \Delta_{n, m}} \eta_{\alpha \beta} u^{\beta}$,

$$
\begin{aligned}
& v_{-a}=\sum_{\substack{\alpha_{1}+\cdots+\alpha_{a}=(a-1) m+a \\
\alpha_{1}, \cdots, \alpha_{a}>0}} u_{-\alpha_{1}} u_{-\alpha_{2}} \cdots \cdots u_{-\alpha_{a}}, \\
& \text { for } 1 \leq a \leq m, \\
& v_{a}=u_{a}+\sum_{b=2}^{n-a-1} \frac{(\alpha+\beta-1) !}{a b !} \sum_{\substack{\alpha_{1}+\cdots+\alpha_{b}=(b-1) n+a+b-1 \\
n-1 \geq \alpha_{1}, \cdots, \alpha_{a} \geq 0}} u_{\alpha_{1}} \cdots \cdots u_{\alpha_{b}}, \\
& \text { for } 0 \leq a \leq n-1, \\
& s=u_{n} .
\end{aligned}
$$

Proof. Through the differentiation of the $\mathrm{W}$ potential by the universal coordinates we obtain for $a, \alpha \geq 0$

$$
\begin{aligned}
\frac{\partial v_{-a}}{\partial u_{-\alpha}} & =\underset{p=\infty}{\operatorname{res}}\left[\phi^{-\alpha}(p-s)^{a-1}\right], \\
\frac{\partial v_{a}}{\partial u_{\alpha}} & =\underset{p=\infty}{\operatorname{res}}\left[\phi^{\alpha} p^{-(a+1)}\right], \\
\frac{\partial v_{-a}}{\partial u_{\alpha}} & =\frac{\partial v_{a}}{\partial u_{-\alpha}}=0 .
\end{aligned}
$$

Further differentiation gives the following recursion relations

$$
\begin{aligned}
\frac{\partial}{\partial u_{-\alpha}} \frac{\partial}{\partial u_{-\beta}} v_{-a} & =(a-1) \frac{\partial}{\partial u_{-(\alpha+\beta-m-1)}} v_{-a+1}, \quad(\alpha, \beta \neq 1), \\
\frac{\partial}{\partial u_{\alpha}} \frac{\partial}{\partial u_{\beta}} v_{a} & =(a+1) \frac{\partial}{\partial u_{\alpha+\beta-n}} v_{a+1} .
\end{aligned}
$$


From (3.7) we have

$$
v_{-1}=u_{-1}, \quad v_{n-1}=u_{n-1}, \quad v_{n-2}=u_{n-2},
$$

and

$$
\frac{\partial v_{-a}}{\partial u_{-1}}=\delta_{a, 1}, \quad \frac{\partial v_{a}}{\partial u_{n}}=0, \quad \text { for }-m \leq a \leq n-1 .
$$

Then the formulae (3.6) can be obtained by solving (3.8) recursively with initial conditions (3.9a).

The most important aspect of the coefficients $u^{i}$ in (3.1) is that they give the conserved densities of the dKP hierarchy. Namely we have:

Theorem 3.4. There exist functions $G^{i j}=G^{i j}(u)$ such that

$$
\frac{\partial u^{i}}{\partial t_{j}}=\frac{\partial}{\partial t_{0}} G^{i j}, \quad i, j \in \mathbf{Z} .
$$

Proof. This can be proved by a general formulation of the dKP hierarchy, which does not depend on the form of $W$ (see Appendix B). Here we give a proof by a direct calculation using the explicit formula of $u^{i}$ given by (3.2): For $i \geq 0$ and any $j \in \mathbf{Z}$ the quantity $G^{i j}$ can be obtained as

$$
\begin{aligned}
\frac{\partial u^{i}}{\partial t_{j}} & =\underset{p=\infty}{\operatorname{res}}\left[\lambda^{i} \frac{\partial \lambda}{\partial t_{j}}\right]=\underset{p=\infty}{\operatorname{res}}\left[\lambda^{i}\left\{Q^{j}, \lambda\right\}\right] \\
& =\underset{p=\infty}{\operatorname{res}}\left[\frac{1}{i+1}\left\{Q^{j}, \lambda^{i+1}\right\}\right]=\frac{\partial}{\partial t_{0}} \underset{p=\infty}{\operatorname{res}}\left[\frac{1}{i+1} \lambda^{i+1} \phi^{j}\right] .
\end{aligned}
$$

For other cases, similar calculations leads to the following explicit formulae for $G^{i j}$ :

$$
\begin{aligned}
& G^{i j}=\frac{1}{i+1} \underset{p=\infty}{\operatorname{res}}\left[\lambda^{i+1} \phi^{j}\right]=\frac{1}{j+1} \underset{p=\infty}{\operatorname{res}}\left[\lambda^{j+1} \phi^{i}\right], \\
& 0 \leq i, j<\infty, \\
& G^{-i-j}=\left\{\begin{array}{lll}
\frac{1}{i-1} \underset{p=s}{\operatorname{ess}}\left[\mu^{i-1} \phi^{-j}\right], & 2 \leq i<\infty, & 1 \leq j<\infty, \\
\frac{1}{j-1} \underset{p=s}{\operatorname{ess}}\left[\mu^{j-1} \phi^{-i}\right], & 1 \leq i<\infty, & 2 \leq j<\infty,
\end{array}\right.
\end{aligned}
$$




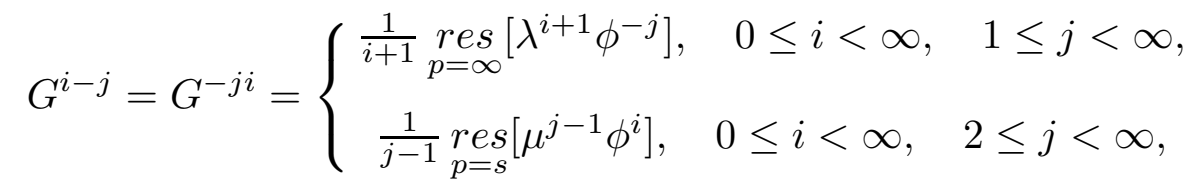

$$
\begin{aligned}
& G^{-1-1}=\underset{p=\infty}{\operatorname{res}}\left[\log \lambda \phi^{-1}\right]+\underset{p=s}{\operatorname{res}}\left[\log \mu \phi^{-1}\right]=\log u_{-m} .
\end{aligned}
$$

These can be more easily obtained by using the formula (4.6) in Proposition 4.3.

Note from (3.11) that $G^{i j}$ are symmetric in the indices, $G^{i j}=G^{j i}$, thereby

$$
\frac{\partial u^{i}}{\partial t_{j}}=\frac{\partial u^{j}}{\partial t_{i}}
$$

We also note from (3.2) that

$$
u^{i}=G^{i 0}
$$

The densities $G^{i j}$ are referred as the generalized Gel'fand-Dikey (GD) potential. As we will show in the next section, these expressions of the generalized GD potentials will lead to the definitions of the N-point functions and the free energy of our TFT.

From Theorem 3.4, we also have:

Corollary 3.5. The generalized GD potential $G^{i j}$ satisfy

$$
\frac{\partial}{\partial t_{k}} G^{i j}=\frac{\partial}{\partial t_{i}} G^{j k}, \quad \text { for } \quad i, j, k \in \mathbf{Z} .
$$

Proof. Taking the derivative of (3.10) with respect to $t_{k}$, and using the commutativity of the flows in $t_{j}$ and $t_{k}$, we obtain

$$
\frac{\partial}{\partial t_{0}} \frac{\partial}{\partial t_{k}} G^{i j}=\frac{\partial}{\partial t_{0}} \frac{\partial}{\partial t_{j}} G^{i k}
$$

This leads to (3.14), except an integration constant which may be taken to be zero.

The Corollary implies that the $G^{i j}$ can be further integrated by both $t_{i}$ and $t_{j}$. This fact will be important in the next section where we define N-point functions from $G^{i j}$ ( Proposition 4.2). 
The formula (3.14) gives a general form of the dKP hierarchy, and is in fact equivalent to the general form of the hierarchy (2.15). For each $k=\alpha_{0} \in \Delta_{n, m}$ in (3.14), one can then define a new set of hierarchy,

$$
\frac{\partial \tilde{u}^{i}}{\partial t_{j}}=\frac{\partial}{\partial t_{\alpha_{0}}} G^{i j}, \quad i, j \in \mathbf{Z}
$$

where the new variable $\tilde{u}^{i}$ is a generalization of $u^{i}$ in (3.13), and is defined as

$$
\tilde{u}^{i}=G^{i \alpha_{0}}
$$

Then the Riemann invariant form of the quasi-linear system (3.15) is given by (2.17). As we will show in Section 4, the primary fields $\tilde{\phi}^{\alpha}$ in (2.18) are related with the generalized universal coordinates $\tilde{u}^{\alpha}$ by the same Legendre transform as in $(3.3)$, i.e.

$$
\frac{\partial W}{\partial \tilde{u}^{\alpha}}=\tilde{\phi}_{\alpha}:=\sum_{\beta \in \Delta_{n, m}} \eta_{\alpha \beta} \tilde{\phi}^{\beta} \quad\left(\bmod W^{\prime}\right)
$$

In (3.15) the dKP hierarchy is defined over the entire phase space of the coupling constants. However, if we restrict the hierarchy only on the small phase space, we obtain:

Proposition 3.6. The dKP hierarchy (3.15) with $i, j \in \Delta_{n, m}$ possesses the following solutions for each $\alpha_{0} \in \Delta_{n, m}$,

$$
\tilde{u}^{\alpha}=\sum_{\beta \in \Delta_{n, m}} \eta^{\alpha \beta} t_{\beta}, \quad \alpha \in \Delta_{n, m}
$$

As a special case with $\alpha_{0}=0$, we have $u_{\alpha}=t_{\alpha}$.

Proof. $\quad$ Note from (3.17) and (3.18) that we have $\partial W / \partial t_{\alpha_{0}}=\partial W / \partial \tilde{u}_{\alpha_{0}}=\tilde{\phi}^{\alpha_{0}}=$ $1\left(\bmod W^{\prime}\right)$. Then calculating (3.15) with (3.18) and finding the same equation as (3.5) in Proposition 3.2 verifies the assertion.

With the solution (3.18), the primary coupling $t_{\alpha_{0}}$ then gives the deformation parameter (cosmological constant) corresponding to the puncture operator $\tilde{\phi}^{\alpha_{0}}=$ $\mathcal{P}$ in the new set of primaries $\left\{\tilde{\phi}^{\alpha}\right\}$ of $(2.18)$, as we have described in Section 2.

It is obvious but important to note that the solution (3.18) do not satisfy the dKP hierarchy for the gravitational couplings $t_{i}$. For each $\alpha_{0}$ the solution of 
(3.18) gives a "flat" solution of the WDVV equation (1.2), and describes the matter sector of TFT at the topological point [DW] (see Section 7). Proposition 3.6 then implies that the dKP hierarchy with a given $\mathrm{W}$ potential admits the same number of flat solutions as of the primaries, that is, $n+m+1$ for $\mathrm{W}$ in (2.1). In order to construct solutions with the gravitational couplings, one needs to solve the dKP hierarchy in the entire phase space. It is however surprising that these solutions can be obtained by solving the dKP hierarchy only in the small phase space. This has been shown in $[\mathrm{KG}]$, and may be considered as the renormalizability of the universal coordinates. This will be further discussed in Section 6 .

\section{$\S 4$. N-point functions}

In this section we give a realization of our TFT by constructing explicit formulae of the N-point functions in the framework of the dKP hierarchy. Let us begin with:

Definition 4.1. A complex function $<\phi^{i_{1}} \cdots \phi^{i_{N}}>$ of $t=\left(t_{i}: i \in \mathbf{Z}\right)$ is a $N$-point function of the fields $\left\{\phi^{i}\right\}$, if there exists a function $F=F(t)$ such that

$$
<\phi^{i_{1}} \cdots \cdots \phi^{i_{N}}>(t)=\frac{\partial}{\partial t_{i_{1}}} \cdots \cdots \frac{\partial}{\partial t_{i_{N}}} F(t) .
$$

Here the function $F(t)$ is called the free energy of a TFT.

From Theorem 3.4 and Corollary 3.5 it is immediate that:

Proposition 4.2. There exists a function $F=F(t)$ such that the generalized $G D$ potential $G^{i j}$ in (3.11) is expressed by

$$
G^{i j}=\frac{\partial}{\partial t_{i}} \frac{\partial}{\partial t_{j}} F
$$

It is then natural to identify $G^{i j}$ to be the 2-point function generated by the free energy $F$,

$$
<\phi^{i} \phi^{j}>=G^{i j}(u) .
$$


This gives a constitutive equation of the 2-point function as a function of the universal coordinates [DW]. Also with (4.3), the variables $\tilde{u}^{i}$ in (3.16) with a fixed $\alpha_{0} \in \Delta_{n, m}$ are expressed by

$$
\tilde{u}^{i}=<\phi^{i} \phi^{\alpha_{0}}>, \quad i \in \mathbf{Z}
$$

Now we define the N-point function of our TFT by differentiating (4.3) with respect to the flow parameters,

$$
<\phi^{i} \phi^{j} \phi^{k_{1}} \cdots \cdots \phi^{k_{N-2}}>=\frac{\partial}{\partial t_{k_{1}}} \cdots \cdots \frac{\partial}{\partial t_{k_{N-2}}}<\phi^{i} \phi^{j}>
$$

In particular one can prove:

Proposition 4.3. The 3-point function defined by (4.5) can be represented by the residue formula

$$
<\phi^{i} \phi^{j} \phi^{k}>=\underset{p \in \operatorname{Ker} W^{\prime}}{r e s}\left[\frac{\phi^{i} \phi^{j} \phi^{k} \frac{\partial W}{\partial t_{0}}}{W^{\prime}}\right]
$$

Proof. With $<\phi^{i} \phi^{j}>=G^{i j}$ of (3.11), it suffices to show

$$
\frac{\partial}{\partial t_{k}}<\phi^{i} \phi^{j}>=\underset{p \in \operatorname{Ker} W^{\prime}}{r e s}\left[\frac{\phi^{i} \phi^{j} \phi^{k} \frac{\partial W}{\partial t_{0}}}{W^{\prime}}\right]
$$

Let us start with the case $i \geq 0, j \leq-2$. By analyitical continuation of the contour in the residue integral we get

$$
\begin{aligned}
\text { the r.h.s } & =\underset{p=\infty}{\operatorname{res}}\left[\frac{\phi^{i} \phi^{j} \frac{\partial W}{\partial t_{k}}}{W^{\prime}}\right]-\underset{p=s}{\operatorname{ees}}\left[\frac{\phi^{i} \phi^{j} \frac{\partial W}{\partial t_{k}}}{W^{\prime}}\right] \\
& =\underset{p=\infty}{\operatorname{res}}\left[\phi^{j} \frac{\partial}{\partial t_{k}}\left(\frac{\lambda^{i+1}}{i+1}\right)\right]-\underset{p=s}{\operatorname{res}}\left[\phi^{i} \frac{\partial}{\partial t_{k}}\left(\frac{\mu^{-j-1}}{j+1}\right)\right] .
\end{aligned}
$$

The second piece becomes

$$
\underset{p=s}{\operatorname{res}}\left[\phi^{i} \frac{\partial}{\partial t_{k}}\left(\frac{\mu^{-j-1}}{j+1}\right)\right]=\underset{p=\infty}{\operatorname{res}}\left[\phi^{i} \frac{\partial}{\partial t_{k}}\left[\frac{\mu^{-j-1}}{j+1}\right]_{-}\right]=\underset{p=\infty}{\operatorname{res}}\left[\frac{\partial}{\partial p}\left(\frac{\lambda^{i+1}}{i+1}\right) \frac{\partial}{\partial t_{k}}\left[\frac{\mu^{-j-1}}{j+1}\right]_{-}\right]
$$


Putting this into (4.8) and using (3.11c) yield the l.h.s. of eq. (4.7). For $j=-1$ we have, with $\phi^{-1}=1 /(p-s)$,

$$
\begin{aligned}
\text { the r.h.s } & =\underset{p=\infty}{\operatorname{res}}\left[\frac{\phi^{i} \frac{\partial W}{\partial t_{k}}}{(p-s) W^{\prime}}\right]-\underset{p=s}{\operatorname{res}}\left[\frac{\phi^{i} \frac{\partial W}{\partial t_{k}}}{(p-s) W^{\prime}}\right] \\
& =\underset{p=\infty}{\operatorname{res}}\left[\frac{\frac{\partial}{\partial t_{k}}\left(\frac{\lambda^{i+1}}{i+1}\right)}{p-s}\right]+\underset{p=s}{\operatorname{res}}\left[\phi^{i} \frac{\frac{\partial s}{\partial t_{k}}}{(p-s)}\right] \\
& \left.=\frac{\partial}{\partial t_{k}}<\phi^{i} \phi^{-1}\right\rangle .
\end{aligned}
$$

For $i=j=-1$ we verify eq. (4.7) by calculating as

$$
\begin{aligned}
\text { the r.h.s. } & =-\underset{p=s}{\operatorname{res}}\left[\frac{\frac{\partial W}{\partial t_{k}}}{(p-s)^{2} W^{\prime}}\right] \\
& =\frac{1}{m} \frac{\partial}{\partial t_{k}} \log v_{-m}=\frac{\partial}{\partial t_{k}}\left\langle\phi^{-1} \phi^{-1}\right\rangle .
\end{aligned}
$$

In the second line we have used the formula (3.6a), i.e. $v_{-m}=\left(u_{-m}\right)^{m}$. For other cases of $i$ and $j$ (4.7) can be shown similarly.

Note here that, if $W$ is a polynomial in $p$, i.e. the $A$-model, the residue in eq. (4.6) can be evaluated at $p=\infty$. We also remark that $W^{\prime}$ is nilpotent in the numerator of the integrand in (4.6), and the residue formula (4.6) is faithful to the ring structure with the ideal $W^{\prime}=0$. With Proposition 4.3, the integrability of the 3-point function is evident, and is equivalent to (3.14) in Corollary 3.5,

$$
\frac{\partial}{\partial t_{i}}<\phi^{j} \phi^{k}>=\frac{\partial}{\partial t_{j}}<\phi^{i} \phi^{k}>=<\phi^{i} \phi^{j} \phi^{k}>.
$$

It should be also noted that the 1-point function obtained by further integration of (4.7) can not be explicitly expressed without giving a specific form of the solutions of the dKP hierarchy (see Section 6).

As was shown in the previous section, the coupling constants $t_{\alpha}$ for $\alpha \in$ $\Delta_{n, m}$ are identified with the universal coordinates $u_{\alpha}$ at a topological limit of TFT (Proposition 3.6). This suggests that we have another type of free energy in terms of the universal coordinates $u^{\alpha}$. Namely we have: 
Proposition 4.4. There exists a function $F_{0}=F_{0}(u)$ such that for $\alpha, \beta \in \Delta_{n, m}$

$$
G^{\alpha \beta}=\frac{\partial}{\partial u_{\alpha}} \frac{\partial}{\partial u_{\beta}} F_{0}
$$

Proof. $\quad$ Note first that the generalized GD potential $G^{\alpha \beta}$ can be considered as the functions of the universal coordinates $u^{\alpha}$ instead of $t_{\alpha}$. From Proposition 3.2 and the explicit form of $G^{\alpha \beta}$, one can easily see that $G^{\alpha \beta}$ can be integrated by both $u^{\alpha}$ and $u^{\beta}$.

Note that the existence of $F_{0}$ results directly from the definition of the universal coordinates, and not as a consequence of the dKP hierarchy. With the more general solution in Proposition 3.6, that is, $\tilde{u}_{\alpha}=<\phi^{\alpha_{0}} \phi_{\alpha}>=t_{\alpha}$ for each $\alpha_{0} \in \Delta_{n, m}$, we have from Corollary 3.5:

Proposition 4.5. There exists a function $F_{\alpha_{0}}=F_{\alpha_{0}}(\tilde{u})$ such that for $\alpha, \beta \in \Delta_{n, m}$

$$
G^{\alpha \beta}=\frac{\partial}{\partial \tilde{u}_{\alpha}} \frac{\partial}{\partial \tilde{u}_{\beta}} F_{\alpha_{0}}
$$

One should note from these propositions that the 2-point function $G^{\alpha \beta}$ is a universal object, that is,

$$
G^{\alpha \beta}=\frac{\partial^{2} F_{0}}{\partial u_{\alpha} \partial u_{\beta}}=\frac{\partial^{2} F_{\alpha_{0}}}{\partial \tilde{u}_{\alpha} \partial \tilde{u}_{\beta}} .
$$

This has been found as a symmetry of the WDVV equation in [Du2].

From Propositions 4.4 and 4.5, we propose:

Definition 4.6. A complex function $<\phi^{\alpha_{1}} \cdots \phi^{\alpha_{N}}>_{0}$ of the universal coordinates $u^{\alpha}$ for $\alpha \in \Delta_{n, m}$ is a universal $N$-point function of the fields $\phi^{\alpha}$, if there exists a function $F_{0}=F_{0}(u)$ such that for $\alpha_{i} \in \Delta_{n, m}$

$$
<\phi^{\alpha_{1}} \cdots \cdots \phi^{\alpha_{N}}>_{0}(u)=\frac{\partial}{\partial u_{\alpha_{1}}} \cdots \cdots \frac{\partial}{\partial u_{\alpha_{N}}} F_{0}(u) .
$$

We call the function $F_{0}$ the universal free energy. In general, one can also define universal $N$-point functions for the fields $\left\{\tilde{\phi}^{\alpha}\right\}$ in (2.18) with the free energy $F_{\alpha_{0}}(\tilde{u})$ as

$$
<\tilde{\phi}^{\alpha_{1}} \cdots \cdots \tilde{\phi}^{\alpha_{N}}>_{\alpha_{0}}(\tilde{u})=\frac{\partial}{\partial \tilde{u}_{\alpha_{1}}} \cdots \cdots \frac{\partial}{\partial \tilde{u}_{\alpha_{N}}} F_{\alpha_{0}}(\tilde{u}) .
$$


Note that $F_{\alpha_{0}}$ gives the free energy $F$ in (4.1) at the topological limit corresponding to the flat solution $\tilde{u}_{\alpha}=t_{\alpha}$. For $\alpha_{0}=0$, we also notice that owing to (3.3) the 2-point functions $G^{\alpha \beta}$ in (3.11) can be further integrated by $u^{\beta}$ and the universal 1-point functions are expressed as the period integrals,

$$
\begin{aligned}
<\phi^{\alpha}>_{0} & =\frac{1}{(\alpha+n+2)(\alpha+1)} \underset{p=\infty}{\operatorname{res}}\left[\lambda^{\alpha+n+2}\right], \quad 0 \leq \alpha \leq n \\
<\phi^{-1}>_{0} & =\underset{p=\infty}{\operatorname{res}}\left[W\left(\log \lambda-\frac{1}{n+1}\right)\right]+\underset{p=s}{\operatorname{res}}\left[W\left(\log \mu-\frac{1}{m}\right)\right], \\
<\phi^{-\alpha}>_{0} & =\frac{1}{(\alpha+m-1)(\alpha-1)} \underset{p=s}{\operatorname{res}}\left[\mu^{\alpha+m-1}\right], \quad 2 \leq \alpha \leq m .
\end{aligned}
$$

These 1-point functions can be extended for all fields $\phi^{i}$ with $i \in \mathbf{Z}$ by integrating $G^{i \alpha}$ with respect to $u_{\alpha}$. With these definitions of the N-point functions, we see the universality of the 2-point functions,

$$
<\phi^{\alpha} \phi^{\beta}>_{0}=<\phi^{\alpha} \phi^{\beta}>=<\tilde{\phi}^{\alpha} \tilde{\phi}^{\beta}>_{\alpha_{0}} .
$$

The universal 3-point function can be also written in the residue formula:

\section{Proposition 4.7.}

$$
<\phi^{\alpha} \phi^{\beta} \phi^{\gamma}>_{0}=\underset{p \in \operatorname{Ker} W^{\prime}}{r e s}\left[\frac{\phi^{\alpha} \phi^{\beta} \phi^{\gamma}}{W^{\prime}}\right]
$$

Proof. Write the r.h.s. as

$$
<\phi^{\alpha} \phi^{\beta} \phi^{\gamma}>_{0}=\underset{p \in \operatorname{Ker} W^{\prime}}{r e s}\left[\frac{\phi^{\alpha} \phi^{\beta} \frac{\partial W}{\partial u_{\gamma}}}{W^{\prime}}\right]
$$

by eq. (3.3). Then eq. (4.18) can be shown similarly as eq. (4.6).

With this Proposition, the metric defined in (3.4) can be expressed by the universal 3 -point functions, i.e. for $\alpha, \beta \in \Delta_{n, m}$,

$$
\eta_{\alpha \beta}=\eta^{\alpha \beta}=<\phi^{\alpha} \phi^{\beta} \phi^{0}>_{0} .
$$


Note that in terms of the flat solution $\tilde{u}_{\alpha}=t_{\alpha}$ obtained in Proposition 3.6 we also have

$$
\eta^{\alpha \beta}=\frac{\partial}{\partial t_{\beta}} G^{\alpha_{0} \alpha}=<\phi^{\alpha} \phi^{\beta} \phi^{\alpha_{0}}>.
$$

The flatness of these 3-point functions is a fundamental property for TFT.

From (4.18), one can define an inner product on the ring $\mathcal{R}$ of (2.10) with a bilinear map, $(\cdot, \cdot)_{0}: \mathcal{R} \times \mathcal{R} \rightarrow \mathbf{C}$,

$$
(\phi, \psi)_{0}:=\underset{p \in \operatorname{Ker} W^{\prime}}{\operatorname{res}}\left[\frac{\phi \psi}{W^{\prime}}\right]=<\phi \psi \phi^{0}>_{0} .
$$

The ring with this inner product $\left\{\mathcal{R},(\cdot, \cdot)_{0}\right\}$ then defines a commutative Frobenius algebra. In particular, the set of primary fields $\left\{\phi^{\alpha}\right\}$ defined in (2.8) gives an orthonormal basis, i.e.

$$
\left(\phi^{\alpha}, \phi_{\beta}\right)_{0}=\delta_{\beta}^{\alpha}=\underset{p \in \operatorname{Ker} W^{\prime}}{\operatorname{res}}\left[\frac{\phi^{\alpha} \phi_{\beta}}{W^{\prime}}\right] .
$$

It follows from (4.22) that the following bilinear map also gives an inner product which makes the fields $\{\tilde{\phi}\}$ in $(2.18)$ to be orthonormal, i.e. for a fixed $\alpha_{0} \in \Delta_{n, m}$,

$$
(\tilde{\phi}, \tilde{\psi})_{\alpha_{0}}:=\underset{p \in \operatorname{Ker} W^{\prime}}{r e s}\left[\frac{\tilde{\phi} \tilde{\psi}\left(\phi^{\alpha_{0}}\right)^{2}}{W^{\prime}}\right] .
$$

Note that $(\phi, \psi)_{0}=(\tilde{\phi}, \tilde{\psi})_{\alpha_{0}}$, i.e. the invariance of the inner product under the change of primaries.

Let us now consider the fusion algebra (2.21) with the universal 3-point functions (4.18). Using (4.21), the structure constants $c_{\gamma}^{\alpha \beta}$ in (2.21) can be written by

$$
c_{\gamma}^{\alpha \beta}=<\phi^{\alpha} \phi^{\beta} \phi_{\gamma}>_{0}=\sum_{\delta \in \Delta_{n, m}}<\phi^{\alpha} \phi^{\beta} \phi^{\delta}>_{0} \eta_{\delta \gamma} .
$$

Then the associativity (1.2c) follows through reducing the following quantity to the universal 3-point functions (4.18),

$$
\underset{p \in \operatorname{Ker} W^{\prime}}{r e s}\left[\frac{\phi^{\alpha} \phi^{\beta} \phi^{\gamma} \phi^{\delta}}{W^{\prime}}\right], \quad \alpha, \beta, \gamma, \delta \in \Delta_{n, m} .
$$

For the case of the dKP hierarchy in the form (2.17), the fusion algebra for $\left\{\tilde{\phi}^{\alpha}\right\}$ is defined in the same way: From (4.23), the structure constants $\tilde{c}_{\gamma}^{\alpha \beta}$ is given by

$$
\tilde{c}_{\gamma}^{\alpha \beta}=<\tilde{\phi}^{\alpha} \tilde{\phi}^{\beta} \tilde{\phi}_{\gamma}>_{\alpha_{0}}=\underset{p \in \operatorname{Ker} W^{\prime}}{\operatorname{res}}\left[\frac{\tilde{\phi}^{\alpha} \tilde{\phi}^{\beta} \tilde{\phi}_{\gamma}\left(\phi^{\alpha_{0}}\right)^{2}}{W^{\prime}}\right]
$$


This also gives a residue formula of the universal 3-point functions for $\left\{\tilde{\phi}^{\alpha}\right\}$, and from (3.17) and (4.17) we have

$$
<\tilde{\phi}^{\alpha} \tilde{\phi}^{\beta} \tilde{\phi}^{\gamma}>_{\alpha_{0}}=\frac{\partial}{\partial \tilde{u}_{\gamma}}<\tilde{\phi}^{\alpha} \tilde{\phi}^{\beta}>_{\alpha_{0}}=\frac{\partial}{\partial \tilde{u}_{\gamma}}<\phi^{\alpha} \phi^{\beta}>_{0}
$$

In particular, we see $\eta^{\alpha \beta}=<\tilde{\phi}^{\alpha} \tilde{\phi}^{\beta} \tilde{\phi}^{\alpha_{0}}>_{\alpha_{0}}$.

We also note

$$
\phi^{\alpha} \phi^{\beta}=\sum_{\gamma \in \Delta_{n, m}} c_{\gamma}^{\alpha \beta} \phi^{\gamma}=\sum_{\gamma \in \Delta_{n, m}} \tilde{c}_{\gamma}^{\alpha \beta} \phi^{\gamma} \phi^{\alpha_{0}} \quad\left(\bmod W^{\prime}\right)
$$

which leads to the linear transformation between the fields $\left\{\phi^{\alpha}\right\}$ and $\left\{\tilde{\phi}^{\alpha}\right\}$,

$$
\phi^{\alpha}=\sum_{\beta \in \Delta_{n, m}} c_{\beta}^{\alpha_{0} \alpha} \tilde{\phi}^{\beta} \quad\left(\bmod W^{\prime}\right)
$$

With $(4.29)$, one can now prove the relation $(3.17), \partial W / \partial \tilde{u}_{\alpha}=\tilde{\phi}^{\alpha}\left(\bmod W^{\prime}\right)$ : Taking the derivative of the $\mathrm{W}$ potential with respect to $u_{\alpha}$, we have

$$
\begin{aligned}
\phi^{\alpha}=\frac{\partial W}{\partial u_{\alpha}} & =\sum_{\beta \in \Delta_{n, m}} \frac{\partial W}{\partial \tilde{u}_{\beta}} \frac{\partial \tilde{u}_{\beta}}{\partial u_{\alpha}}=\sum_{\beta \in \Delta_{n, m}} \frac{\partial W}{\partial \tilde{u}_{\beta}}<\phi^{\alpha_{0}} \phi^{\alpha} \phi_{\beta}>_{0} \\
& =\sum_{\beta \in \Delta_{n, m}} \frac{\partial W}{\partial \tilde{u}^{\beta}} c_{\beta}^{\alpha_{0} \alpha}\left(\bmod W^{\prime}\right) .
\end{aligned}
$$

Using (4.29) then leads to the relation (3.17).

Before closing this section, we remark as a corollary to Proposition 4.5 that the dKP hierarchy in (3.15) for the entire phase space can be put into a hamiltonian form,

$$
\frac{\partial \tilde{u}^{\alpha}}{\partial t_{i}}=\frac{\partial}{\partial t_{\alpha_{0}}} \frac{\partial \tilde{H}^{i}}{\partial \tilde{u}_{\alpha}}=\frac{\partial}{\partial t_{\alpha_{0}}} \sum_{\beta \in \Delta_{n, m}} \eta^{\alpha \beta} \frac{\partial \tilde{H}^{i}}{\partial \tilde{u}^{\beta}}, \quad i \in \mathbf{Z}
$$

where the hamiltonian function $\tilde{H}^{i}$ is given by the 1-point function $<\tilde{\phi}^{i}>_{\alpha_{0}}$ defined in (4.15b). Note here that the index $i$ in the 1-point function is extended to $i \in \mathbf{Z}$, as explained below (4.16).

\section{$\S 5$. The topological recursion relation}


In addition to eqs. (1.2), the TFT's also satisfy the so-called "topological recursion relation" which gives a recursion for the 3-point functions of the descendants. In this section we show that the dKP hierarchy (2.4) also provides this important property.

Let us first note from $\partial W / \partial u_{0}=\phi^{0}=1\left(\bmod W^{\prime}\right)$ that

$$
\begin{aligned}
& \lambda^{i+1}=\lambda^{i+1} \frac{\partial W}{\partial u_{0}}=\frac{\partial}{\partial u_{0}}\left(\frac{\lambda^{(n+1)+i+1}}{(n+1)+i+1}\right), \quad \text { for } i \geq 0, \\
& \mu^{j-1}=\mu^{j-1} \frac{\partial W}{\partial u_{0}}=\frac{\partial}{\partial u_{0}}\left(\frac{\mu^{m+j-1}}{m+j-1}\right), \quad \text { for } j \geq 2 .
\end{aligned}
$$

Setting $i=N(n+1)+\alpha$ and $j=N m+\beta$ with an integer $N \geq 0$ and $0 \leq \alpha \leq$ $n, 2 \leq \beta \leq m$, we have the recursion relation among $\phi^{i}$, except $i=-1$,

$$
\begin{aligned}
\phi^{N(n+1)+\alpha} & =\frac{1}{N(n+1)+\alpha+1} \frac{\partial}{\partial u_{0}} \phi^{(N+1)(n+1)+\alpha} \\
\phi^{-N m-\beta} & =\frac{1}{N m+\beta-1} \frac{\partial}{\partial u_{0}} \phi^{-(N+1) m-\beta} .
\end{aligned}
$$

Then we define the fields $\sigma_{N}\left(\phi^{\alpha}\right)$ for $N \geq 1$, the descendant fields, except $\alpha=-1$,

$$
\sigma_{N}\left(\phi^{\alpha}\right):=\frac{\partial Q_{N}^{\alpha}}{\partial p}=\left\{\begin{array}{cc}
c_{N, \alpha} \phi^{N(n+1)+\alpha}, & 0 \leq \alpha \leq n, \\
d_{N, \alpha} \phi^{-N m-|\alpha|}, & -m \leq \alpha \leq-2,
\end{array}\right.
$$

where the normalization constants $c_{N, \alpha}$ and $d_{N, \alpha}$ are defined by

$$
\begin{aligned}
& c_{N, \alpha}=[(\alpha+1)(\alpha+1+n+1) \cdots \cdots(\alpha+1+(N-1)(n+1))]^{-1}, \\
& d_{N, \alpha}=[(|\alpha|-1)(|\alpha|-1+m) \cdots \cdots(|\alpha|-1+(N-1) m)]^{-1} .
\end{aligned}
$$

For the case of $\alpha=-1$, the descendant field can be also defined in the same way. Namely we have, modulo $W^{\prime}$,

$$
\begin{aligned}
& W^{N}\left(\log \lambda-c_{N}\right)=\frac{1}{N+1} \frac{\partial}{\partial u_{0}}\left(W^{N+1}\left(\log \lambda-c_{N+1}\right)\right) \\
& W^{N}\left(\log \mu-d_{N}\right)=\frac{1}{N+1} \frac{\partial}{\partial u_{0}}\left(W^{N+1}\left(\log \mu-d_{N+1}\right)\right),
\end{aligned}
$$


where the constants $c_{N}$ and $d_{N}$ are defined by

$$
\begin{aligned}
& c_{N}=\frac{1}{n+1} \sum_{l=1}^{N} \frac{1}{l}, \\
& d_{N}=\frac{1}{m} \sum_{l=1}^{N} \frac{1}{l},
\end{aligned}
$$

for $N \geq 1$ and $c_{0}=d_{0}=0$. The descendant fields $\sigma_{N}\left(\phi^{-1}\right)$ are then defined by

$$
\begin{aligned}
\sigma_{N}\left(\phi^{-1}\right): & =\frac{\partial Q_{N}^{-1}}{\partial p} \\
& =\frac{\partial}{\partial p}\left(\left[\frac{W^{N}}{N !}\left(\log \lambda-c_{N}\right)\right]_{+}-\left[\frac{W^{N}}{N !}\left(\log \mu-d_{N}\right)\right]_{-}\right)
\end{aligned}
$$

In Appendix A, we give the explicit formula of (5.7), and show that $\sigma_{N}\left(\phi^{-1}\right)$ is defined as an element of the ring $\mathcal{R}$ of (2.10). The compatibility of this field with others can be also shown in the similar way as in Lemma 2.1. Eq.(5.7) gives a precise definition and generalization of the descendant fields of $\phi^{-1}$ found in $[\mathrm{EY}]$. With these definitions (5.3) and (5.7), eqs.(5.2) and (5.5) lead to a recursion relation,

$$
\sigma_{N-1}\left(\phi^{\alpha}\right)=\frac{\partial}{\partial u_{0}} \sigma_{N}\left(\phi^{\alpha}\right), \quad\left(\bmod W^{\prime}\right) .
$$

Correspondingly to those descendant fields, we also define the gravitational coupling constants $t_{N, \alpha}$ as

$$
t_{N, \alpha}=\left\{\begin{array}{cl}
c_{N, \alpha}^{-1} t_{N(n+1)+\alpha}, & 0 \leq \alpha \leq n, \\
t_{N,-1}, & \alpha=-1 \\
d_{N, \alpha}^{-1} t_{-N m-|\alpha|}, & -m \leq \alpha \leq-2,
\end{array}\right.
$$

Thus the gravitational descendants are constructed entirely from the primary (matter) fields alone, similar to the case of the minimal model [Lo, EKYY, EYY1].

With the definitions of $Q_{N}^{-1}$ in (5.7), and $t_{N,-1}$ in (5.9), one can extend the 3 -point function in Proposition 4.3 to include the field $\sigma_{N}\left(\phi^{-1}\right)$. In particular, following the calculations in Appendix A and in the proof of Proposition 4.3, we find the formula for the 2-point function with $\sigma_{N}\left(\phi^{-1}\right)$,

$$
<\sigma_{N}\left(\phi^{-1}\right) \phi^{i}>=\underset{p=\infty}{\operatorname{res}}\left[\frac{W^{N}}{N !}\left(\log \lambda-c_{N}\right) \phi^{i}\right]+\underset{p=s}{\operatorname{exs}}\left[\frac{W^{N}}{N !}\left(\log \mu-d_{N}\right) \phi^{i}\right] .
$$


As shown in (4.14), the 2-point function $<\sigma_{N}\left(\phi^{-1}\right) \phi^{\alpha}>$ can be further integrated by $u_{\alpha}$, and the flow $\partial u^{\alpha} / \partial t_{N,-1}$ is expressed in the hamiltonian form of (4.34) with $H_{N}^{-1}$,

$$
\begin{aligned}
& H_{N}^{-1}=<\sigma_{N}\left(\phi^{-1}\right)>_{0} \\
& =\underset{p=\infty}{\operatorname{res}}\left[\frac{W^{N+1}}{(N+1) !}\left(\log \lambda-c_{N+1}\right)\right]+\underset{p=s}{\operatorname{res}}\left[\frac{W^{N+1}}{(N+1) !}\left(\log \mu-d_{N+1}\right)\right] .
\end{aligned}
$$

By the ideal $W^{\prime}=0$, we have the extended relation of $(2.9)$,

$$
\sigma_{N}\left(\phi^{n}\right)=\sigma_{N}\left(\phi^{-(m+1)}\right) \quad\left(\bmod W^{\prime}\right), \quad N \geq 0
$$

with the identifications $\sigma_{0}\left(\phi^{\alpha}\right)=\phi^{\alpha}, c_{0, \alpha}=d_{0, \alpha}=1$ for any $\alpha \in \Delta_{n, m}$, and $\sigma_{N}\left(\phi^{-(m+1)}\right)=\phi^{-(N+1) m-1} / N ! m^{N}$. This implies that the solutions of the dKP hierarchy (2.14) have the form with $t_{0, \alpha}:=t_{\alpha}$,

$$
u^{\alpha}=u^{\alpha}\left(t_{N, \beta}: t_{N, n}+t_{N,-(m+1)} \text {, for each } N \geq 0, \text { and } \beta \in \Delta_{n, m}\right) .
$$

where $t_{N,-(m+1)}$ is the flow parameter corresponding to the field $\sigma_{N}\left(\phi^{-(m+1)}\right)$, i.e. $t_{N,-(m+1)}=N ! m^{N} t_{-(N+1) m-1}$. Note that these $\sigma_{N}\left(\phi^{-(m+1)}\right)$, and $t_{N,-(m+1)}$ were readily excluded from the defining relations (5.3) and (5.9). However, because of (5.13) we identify $t_{N, n}$ with $t_{N, n}+t_{N,-(m+1)}$. With these notations one obtains the main theorem of this section:

Theorem 5.1. For each primary $\phi^{\alpha}$, we have the topological recursion relation for the 3-point functions,

$$
<\sigma_{N}\left(\phi^{\alpha}\right) A B>=\sum_{\beta \in \Delta_{n, m}}<\sigma_{N-1}\left(\phi^{\alpha}\right) \phi^{\beta}><\phi_{\beta} A B>
$$

for any $A, B \in \mathbf{C}\left[p,(p-s)^{-1}\right]$ and $N \geq 1$.

To prove this theorem we need:

Lemma 5.2. The descendants can be decomposed into the primaries, i.e.,

$$
\sigma_{N}\left(\phi^{\alpha}\right)=\sum_{\beta \in \Delta_{n, m}}<\sigma_{N-1}\left(\phi^{\alpha}\right) \phi^{\beta}>\phi_{\beta} \quad\left(\bmod W^{\prime}\right)
$$


Proof. From the orthonormality (4.22), we note that eq. (5.15) is equivalent to

$$
\begin{aligned}
<\sigma_{N-1}\left(\phi^{\alpha}\right) \phi^{\beta}> & =<\sigma_{N}\left(\phi^{\alpha}\right) \phi^{\beta} \phi^{0}>_{0} \\
& =\underset{p \in \operatorname{Kes} W^{\prime}}{\operatorname{ees}}\left[\frac{\sigma_{N}\left(\phi^{\alpha}\right) \phi^{\beta}}{W^{\prime}}\right] .
\end{aligned}
$$

The function in the residue has a pole at either $p=\infty$ or $p=s$ in addition to those at the roots of $W^{\prime}=0$. Therefore we evaluate (5.16) for the case of $\alpha \neq-1$

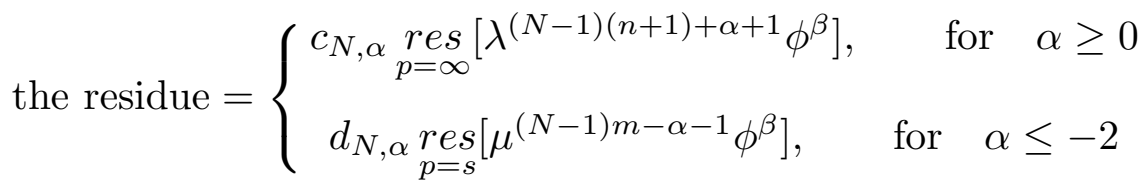

which gives, with (3.11), the l.h.s. of (5.16). For the case of $\alpha=-1$, we have

$$
\begin{aligned}
& <\sigma_{N}\left(\phi^{-1}\right) \phi^{\beta} \phi^{0}>_{0}=\underset{p \in \operatorname{Ker} W^{\prime}}{\operatorname{res}}\left[\frac{\sigma_{N}\left(\phi^{-1}\right) \phi^{\beta} \phi^{0}}{W^{\prime}}\right] \\
& =\underset{p=\infty}{\operatorname{res}}\left[\frac{W^{N-1}}{(N-1) !}\left(\log \lambda-c_{N-1}\right) \phi^{\beta}\right]+\underset{p=s}{\operatorname{res}}\left[\frac{W^{N-1}}{(N-1) !}\left(\log \mu-d_{N-1}\right) \phi^{\beta}\right] \\
& =<\sigma_{N-1}\left(\phi^{-1}\right) \phi^{\beta}>.
\end{aligned}
$$

Then the proof of Theorem 5.1 is straightforward with the residue formula of the three point functions (4.6). From (5.16), we also note the recursion relation for the hamiltonian functions $H_{N}^{\alpha}:=<\sigma_{N}\left(\phi^{\alpha}\right)>_{0}$ for all $\alpha \in \Delta_{n, m}$ and $N \geq 1$,

$$
\frac{\partial H_{N}^{\alpha}}{\partial u_{0}}=H_{N-1}^{\alpha} \text {. }
$$

\section{$\S 6$. The string equations}

Here we derive the "string equation" as the solution of the dKP hierarchy (3.10), and give an explicit scheme to construct the corresponding free energy. The main result in this section is to show that all the effects of the gravitational couplings to the constitutive equations (2-point functions) can be described in the small phase space alone by renormalizing the primary couplings, that is, the renormalizability of the solutions of our TFT. Let us first note from Theorem 5.1: 
Corollary 6.1. The $d K P$ hierarchy (3.10) for the gravitational couplings $t_{N, \beta}$ can be decomposed into the flows for the primaries with $t_{\gamma}$,

$$
\frac{\partial u^{\alpha}}{\partial t_{N, \beta}}=\sum_{\gamma \in \Delta_{n, m}}<\sigma_{N-1}\left(\phi^{\beta}\right) \phi_{\gamma}>\frac{\partial u^{\alpha}}{\partial t_{\gamma}}, \quad \alpha, \beta \in \Delta_{n, m}
$$

where the 2-point funtion $<\sigma_{N-1}\left(\phi^{\beta}\right) \phi_{\gamma}>$ is a function of $u^{\alpha}$ given by (4.3).

From Corollary 6.1 one can obtain:

Theorem 6.2. $[K G]$ The solution of the dKP hierarchy can be expressed by

$$
u^{\alpha}\left(t_{j}: j \in \mathbf{Z}\right)=\hat{u}^{\alpha}\left(\hat{t}_{\beta}: \beta \in \Delta_{n, m}\right), \quad \text { for } \alpha \in \Delta_{n, m}
$$

where $\hat{u}^{\alpha}$ and $\hat{t}^{\beta}$ are given by for all $\alpha, \beta \in \Delta_{n, m}$

$$
\begin{aligned}
& \hat{u}^{\alpha}\left(t_{\gamma}: \gamma \in \Delta_{n, m}\right)=u^{\alpha}\left(\cdots, 0, t_{-m}, \cdots, t_{n}, 0, \cdots\right) \\
& \hat{t}_{\beta}=t_{\beta}+\sum_{\substack{\alpha \in \Delta_{n}, m \\
N \geq 1}}<\sigma_{N-1}\left(\phi^{\alpha}\right) \phi_{\beta}>t_{N, \alpha}
\end{aligned}
$$

Proof. Eq. (6.1) can be expressed in the invariant form of the vector field $X_{N}^{\beta}$,

$$
X_{N}^{\beta} u^{\alpha}=0 \quad \text { for } \alpha, \beta \in \Delta_{n, m}, \text { and } N \geq 1 \text {, }
$$

with

$$
X_{N}^{\beta}:=\frac{\partial}{\partial t_{N, \beta}}-\sum_{\gamma \in \Delta_{n, m}}<\sigma_{N-1}\left(\phi^{\beta}\right) \phi_{\gamma}>\frac{\partial}{\partial t_{\gamma}}
$$

This implies that each $u^{\alpha}$ is constant along the characteristic, which are straight lines, given by, for $\alpha, \beta \in \Delta_{n, m}$ and $N \geq 1$,

$$
\frac{d t_{N, \beta}}{-1}=\frac{d t_{\alpha}}{<\sigma_{N-1}\left(\phi^{\beta}\right) \phi_{\alpha}>}
$$

The integrals of eq. (6.6) are

$$
\hat{t}_{\alpha}=t_{\alpha}+\sum_{\beta \in \Delta_{n, m}}<\sigma_{N-1}\left(\phi^{\beta}\right) \phi_{\alpha}>t_{N, \beta}
$$

which gives eq. (6.3b) on taking sum over $N \geq 1$. Here $\hat{t}_{\alpha}$ gives the initial position of the characteristics at $t_{N, \beta}=0$ for all $N \geq 1$. Note then that $\hat{u}^{\alpha}$ in $(6.3 \mathrm{a})$ are the 
solutions of dKP hierarchy in the small phase space labeled by $\left\{\hat{t}_{\alpha}: \alpha \in \Delta_{n, m}\right\}$. This completes the proof.

This theorem implies that the solution of the dKP hierarchy is completely determined by that in the small phase space, $\hat{u}^{\alpha}$. To be concrete, we first note that eq. (6.3b) may be written in the following form, which gives the string equation of our TFT (see the end of this section),

$$
0=\tilde{t}_{\alpha}+\sum_{\substack{\beta \in \Delta_{n}, m \\ N \geq 1}}<\sigma_{N-1}\left(\phi^{\beta}\right) \phi_{\alpha}>\tilde{t}_{N, \beta},
$$

by shifting the couplings with arbitrary constants $C_{N, \beta}$ as

$$
\tilde{t}_{N, \beta}=t_{N, \beta}+C_{N, \beta}, \quad \text { for } \quad N \geq 1,
$$

and by imposing the relations,

$$
-\sum_{\substack{\beta \in \Delta_{n}, m \\ N \geq 1}}<\sigma_{N-1}\left(\phi^{\beta}\right) \phi_{\alpha}>C_{N, \beta}=\hat{t}_{\alpha} .
$$

Note here that the dKP hierarchy is translationally invariant in the couplings, that is, the solution can be written in the shifted variables $\tilde{t}_{N, \alpha}$. A solution $\hat{u}^{\alpha}\left(\hat{t}_{\gamma}\right)$ of the $\mathrm{dKP}$ hierarchy in the small phase space is then given by solving the algebraic equations (6.9b). For example, by the choice of $C_{N, \beta}=-\delta_{N, 1} \delta_{\beta, \alpha_{0}}$, this equation coincides with (3.18), so that the dKP hierarchy provides a flat solution of our TFT. All the flat solutions are indeed obtained from this choice of the constants $C_{N, \beta}$. The solution in the large phase space is given by merely writing $\hat{t}_{\gamma}$ in $\hat{u}^{\alpha}\left(\hat{t}_{\gamma}\right)$ by $(6.3 \mathrm{~b})$. This is an implicit solution called the hodogragh solution, which still includes the function $\hat{u}^{\alpha}$ in the r.h.s. of $(6.3 \mathrm{~b})$. (See below for the construction of explicit solutions.) Thus depending on the values of $C_{N, \beta}$ one can construct infinitely many solutions of the dKP hierarchy in the entire phase space. Physically speaking, a choice of $C_{N, \beta}$ amounts to considering a TFT in the small phase space where the gravitational couplings take the fixed values,

$$
\tilde{t}_{N, \beta}=C_{N, \beta}, \quad N \geq 1, \quad \beta \in \Delta_{n, m},
$$

and the TFT in the large phase space is obtained as a perturbation from this gravity background. 
The physical meaning of (6.2) is that all the gravitational effects in the universal coordinates $u^{\alpha}(t)$, consequently in the 2-point function $\left\langle\phi^{\alpha} \phi^{\beta}>\right.$ are renormalized into the primary couplings $\hat{t}_{\alpha}$ by $(6.3 \mathrm{~b})$. This renormalizability of the TFT can be most properly seen by writing the action (2.13) as

$$
S=S_{0}+\sum_{\substack{\alpha \in \Delta_{n}, m \\ N \geq 0}} t_{N, \alpha} \sigma_{N}\left(\phi^{\alpha}\right)=S_{0}+\sum_{\alpha \in \Delta_{n, m}} \hat{t}_{\alpha} \phi^{\alpha} \quad\left(\bmod W^{\prime}\right) .
$$

This follws from Lemma 5.2 for the decomposition of the descendants. By (6.3b) and $(6.9 \mathrm{~b})$ we obtain the string equation in the generalized form,

$$
-\sum_{\substack{\beta \in \Delta_{n}, m \\ N \geq 1}}<\sigma_{N-1}\left(\phi^{\beta}\right) \phi_{\alpha}>C_{N, \beta}=t_{\alpha}+\sum_{\substack{\beta \in \Delta_{n}, m \\ N \geq 1}}<\sigma_{N-1}\left(\phi^{\beta}\right) \phi_{\alpha}>t_{N, \beta} .
$$

Here the 2-point functions are the known function of $u_{\alpha}$ by the explicit forms (3.11) and (5.10), thereby (6.12) gives an implicit solution of the dKP hierarchy, the generalized hodograph solution. In order to solve (6.12) explicitly, we employ a perturbation method, assuming the gravitational couplings $t_{N, \alpha}$ to be small, where the leading solution is given by $(6.9 \mathrm{~b})$ with $\hat{t}_{\alpha}=t_{\alpha}$, i.e. $t_{N, \alpha}=0$ for $N \geq 1$. Thus one obtains the solution $u^{\alpha}\left(t_{i}\right)$ as a formal series in the gravitational couplings $t_{N, \alpha}$. This is the well-known renormalization procedure in the quantum field theory. For example, in the case where $C_{N, \beta}=-\delta_{N, 1} \delta_{\beta, 0}$, eq. (6.9b) gives the simplest solution

$$
\hat{u}_{\alpha}=\hat{t}_{\alpha}
$$

The string equation (6.12) then becomes

$$
u_{\alpha}=t_{\alpha}+\sum_{\substack{\beta \in \Delta_{n}, m \\ N \geq 1}}<\sigma_{N-1}\left(\phi^{\beta}\right) \phi_{\alpha}>t_{N, \beta}
$$

which was discussed in [DW, EYY2]. The above mentioned renormalization can be easily carried out in this case.

Let us now give an explicit form of the free energy $F$ of our TFT. Based on the string equation (6.12) we have: 
Theorem 6.2. [Du1, Kr1, TT2] The free energy corresponding to eq. (6.9b) is given by

$$
\begin{aligned}
& F\left(t_{N, \alpha}: \alpha \in \Delta_{n, m}, N \geq 0\right) \\
& =\frac{1}{2} \sum_{\substack{\alpha, \beta \in \Delta_{n, m}, N, M \geq 0}} \tilde{t}_{N, \alpha} \tilde{t}_{M, \beta}<\sigma_{N}\left(\phi^{\alpha}\right) \sigma_{M}\left(\phi^{\beta}\right)>(t),
\end{aligned}
$$

where $\tilde{t}_{0, \alpha}=t_{\alpha}$.

Proof. By multiplying (6.8) by $\left\langle\phi^{\alpha} A B>\right.$ with any fields $A, B$ and using the topological recursion relation (5.15), the string equation (6.8) becomes

$$
0=\sum_{\substack{\beta \in \Delta_{n, m} \\ N \geq 0}} \tilde{t}_{N, \beta}<\sigma_{N}\left(\phi^{\beta}\right) A B>=\sum_{\substack{\beta \in \Delta_{n, m} \\ N \geq 0}} \tilde{t}_{N, \beta} \frac{\partial}{\partial t_{N, \beta}}<A B>.
$$

This shows that the 2-point function is a homogenious function of degree zero in $t_{N, \beta}$, so that the free energy is of degree two, that is, with an Euler operator $\mathcal{E}[\cdot]$,

$$
\mathcal{E}[F]:=\sum_{\substack{\beta \in \Delta_{n, m}, N \geq 0}} \tilde{t}_{N, \beta} \frac{\partial}{\partial t_{N, \beta}} F=2 F .
$$

The formula (6.14) is then obtained by applying the Euler operator once again to (6.16).

One should again note that the 2-point functions $<\phi^{i} \phi^{j}>$ in eq. (6.14) are the explicitly given quantities in terms of the universal coordinates $\left\{u^{\alpha}\right\}$, which are the solutions of the dKP hierarchy. We also note that eq. (6.14) can be reduced to the free energy on the small phase space with $t_{N, \alpha}=0$ for $N \geq 1$,

$$
\begin{aligned}
\hat{F}\left(t_{\gamma}: \gamma \in \Delta_{n, m}\right) & =\frac{1}{2} \sum_{\alpha, \beta \in \Delta_{n, m}} t_{\alpha} t_{\beta}<\phi^{\alpha} \phi^{\beta}>+\sum_{\substack{\alpha, \beta \in \Delta_{n, m} \\
N \geq 1}} C_{N, \alpha} t_{\beta}<\sigma_{N}\left(\phi^{\alpha}\right) \phi^{\beta}> \\
& +\frac{1}{2} \sum_{\substack{\alpha, \beta \in \Delta_{n, m} \\
N, M \geq 1}} C_{N, \alpha} C_{M, \beta}<\sigma_{N}\left(\phi^{\alpha}\right) \sigma_{M}\left(\phi^{\beta}\right)>.
\end{aligned}
$$

In the section 8 , we discuss several examples of LG potentials, and give the explicit formulae of the free energy. 
As a final remark we note that the string equation (6.8) can be put in the form,

$$
L_{-1} Z:=\left(\sum_{\substack{\beta \in \Delta_{n, m} \\ N \geq 1}} \tilde{t}_{N, \beta} \frac{\partial}{\partial t_{N-1, \beta}}+\frac{1}{2} \sum_{\alpha, \beta \in \Delta_{n, m}} \eta^{\alpha \beta} \tilde{t}_{\alpha} \tilde{t}_{\beta}\right) Z=0,
$$

where $Z$ is the partition function defined by $F=\log Z$. This would be generalized to the Virasoro constraints $L_{i} Z=0$ for $i \geq-1$. However there is a subtle problem to determine the Virasoro operators with $i \geq 0$ in a TFT having a scale-violation like some of our TFT's. In the recent paper [EHY], this was discussed for the $C P^{1}$-model, and the explicit forms of the Virasoro operators were obtained. It is interesting to investigate this issue for our general model.

\section{$\S 7$. Critical phenomena}

In this section we discuss the critical behavior of the TFT coupled to the gravity based on the string equation (6.12). This corresponds to studying that of a matrix model in the genus-zero (classical) limit, which would obey a constrained $\mathrm{KP}$ hierarchy with the $\mathrm{W}$ potential in a pseud-differential form.

We call our TFT with the rational potential (2.1) as $W_{n, m}$-model. For example, $W_{n, 0}$ gives the $A_{n}$-model, and $W_{2 n+1,2}$ with the $\mathbf{Z}_{2}$-symmetry, where the deformation variables are constrained by $s=0$ and $v_{2 \alpha+1}=0$ for $-1 \leq \alpha \leq n-1$, gives the $D_{n}$-model. Also, $W_{2 n+1,2 m}$ with the $\mathbf{Z}_{2}$-symmetry is a natural extension of the latter $[\mathrm{T}]$. ( The truncation by the $\mathbf{Z}_{a}$-symmetry with $a \geq 3$ does not make sense, since the flat metric is vanishing for the primaries given by $\phi^{\alpha}$, where $\alpha$ is the $a$-multiple. In this regard there is a misstatement in our previous paper $[\mathrm{AK}]$.$) As is clear from the free energy (6.14)$, the $W_{n, m}$-model with non-zero $m$ in general has a scaling violation due to the log-term. The above models are all of the types which do not have such a violation in our TFT.

We now study critical phenomena of the $W_{n, m}$-model by scaling to the 
special gravity background given by (6.10),

$$
\tilde{t}_{N, \beta}=-\delta_{N, N_{0}} \delta_{\beta, \beta_{0}} \quad \text { for some } \beta_{0}, N_{0} \geq 1
$$

This amounts to studying the dKP hierarchy in the small phase space with $C_{N, \beta}=$ $-\delta_{N, N_{0}} \delta_{\beta, \beta_{0}}$. The solution of the hierarchy is then given by the string equation (6.12), i.e.,

$$
<\sigma_{N_{0}-1}\left(\phi^{\beta_{0}}\right) \phi_{\alpha}>=t_{\alpha}
$$

For the scaling models, we find the critical behavior of the free energy,

$$
F \longrightarrow \epsilon^{2-\gamma_{\text {string }}} F, \quad \text { as } \quad t_{\alpha} \longrightarrow \epsilon^{1-\gamma_{\alpha}} t_{\alpha} .
$$

Here $\epsilon$ is a (dimensionless) scaling parameter, $\gamma_{\text {string }}$ is called the string susceptibility [FGZ], and $\gamma_{\alpha}$ is the critical exponent of the primary $\tilde{\phi}^{\alpha}$ following from (2.17), i.e.

$$
\tilde{\phi}^{\alpha} \longrightarrow \epsilon^{\gamma_{\alpha}} \tilde{\phi}^{\alpha}
$$

Since $\tilde{\phi}^{\alpha_{0}}=\mathcal{P}(=1)$ the puncture operator, we take $\gamma_{\alpha_{0}}=0$. Hence the dimension of $t_{\alpha_{0}}$ equals to 1 , and $t_{\alpha_{0}}=t_{\mathcal{P}}$, the cosmological constant. The values of $\gamma_{\text {string }}$ and $\gamma_{\alpha}$ can be computed by a dimensional analysis of the string equation (7.1). (Make use of the case $\alpha=\alpha_{0}$ to fix the dimention of $\lambda$ and $\mu$.) Here we give only the results:

a) Case with $\alpha_{0} \geq 0$ :

$$
\begin{array}{rlrl}
\gamma_{\text {string }} & =-\frac{2}{d_{+ \pm}}, & & \\
\gamma_{\alpha} & =\frac{\alpha-\alpha_{0}}{d_{+ \pm}}, & \text {for } \quad \alpha \geq 0 \\
\gamma_{\alpha} & =-\frac{(\alpha+1) \frac{n+1}{m}+\alpha_{0}+1}{d_{+ \pm}}, & \text {for } \quad \alpha<0
\end{array}
$$

where

$$
\begin{array}{ll}
d_{++}=N_{0}(n+1)-\alpha_{0}+\beta_{0}, & \text { for } \quad \beta_{0} \geq 0 \\
d_{+-}=N_{0}(n+1)-\alpha_{0}-1-\left(\beta_{0}+1\right) \frac{n+1}{m}, & \text { for } \quad \beta_{0}<0 .
\end{array}
$$


b) Case with $\alpha_{0}<0$ :

$$
\begin{array}{rlrl}
\gamma_{\text {string }} & =-\frac{2}{d_{- \pm}}, & & \\
\gamma_{\alpha} & =\frac{(\alpha+1) \frac{m}{n+1}+\alpha_{0}+1}{d_{- \pm}}, & \text {for } \quad \alpha \geq 0, \\
\gamma_{\alpha} & =-\frac{\alpha-\alpha_{0}}{d_{- \pm}}, & \text {for } \quad \alpha<0,
\end{array}
$$

where

$$
\begin{array}{ll}
d_{-+}=N_{0} m+\alpha_{0}+1+\left(\beta_{0}+1\right) \frac{m}{n+1}, & \text { for } \quad \beta_{0} \geq 0, \\
d_{--}=N_{0} m+\alpha_{0}-\beta_{0}, & \text { for } \quad \beta_{0}<0 .
\end{array}
$$

For the $W_{n, 0}\left(A_{n}\right)$-model these results are well known in the critical analysis of matrix models [Do, GGPZ, FK, FKN, DVV2]. In fact the critical $W_{n, 0}$-model is obtained as the genus-zero limit of the double scaled $(p-1)$-matrix model at the $q$ th criticality in which

$$
p-1=n, \quad q-1=\left(N_{0}-1\right)(n+1)+\beta_{0} .
$$

The latter model is identified with the $(p, q)$ minimal model coupled to the gravity. Among the minimal ones the $(n+1, n+2)$ model, for $N_{0}=2$ and $\beta_{0}=0$, falls into the unitary series, of which the central charge is given by $1-\frac{6}{(n+1)(n+2)}$ in the gravitationless limit [KPZ, DK, Da]. The $(n+1, q)$ models with $1 \leq q \leq n+1$ correspond to the topological limits of the $W_{n, 0}$-model.

For the $W_{2 \nu+1,2 \mu}$-model with the $\mathbf{Z}_{2}$-symmetry these results should be understood with the following parametrization of the indices,

$$
\begin{aligned}
& n=2 \nu+1, \quad m=2 \mu, \quad \text { and }, \\
& \alpha=2 a, \quad \text { for } a \in \Delta_{\nu, \mu} .
\end{aligned}
$$

An interesting observation about the models of this type is that there is a symmetry in the critical exponents given by (7.4) and (7.5) under the simultaneous interchange

$$
\begin{aligned}
& n \rightarrow m-1, \quad m \rightarrow n+1, \quad \text { and } \\
& \alpha+1 \rightarrow-(\alpha+1) .
\end{aligned}
$$

This implies that the $W_{2 \nu+1,2 \mu}$-model in the gravity background given by $\tilde{t}_{N, \beta}=$ $-\delta_{N, N_{0}} \delta_{\beta, \beta_{0}}$ has the same critical behavior as the $W_{2 \mu-1,2 \nu+2}$-model in the background $\tilde{t}_{N, \beta}=-\delta_{N, N_{0}} \delta_{\beta,-\left(\beta_{0}+2\right)}$, if the primary coupling $t_{\alpha}, \alpha \in \Delta_{2 \nu, 2 \mu}$ in the 
former is identified with $t_{-(\alpha+2)},-(\alpha+2) \in \Delta_{2 \mu-2,2 \nu+2}$ in the latter. This symmetry holds for any $N_{0}(\geq 1)$. Hence both models are considered to be physically equivalent even when the gravity is turned on, by generalizing the identification of the primary couplings for the descendant couplings. Thus there exists an equivalent pair in the $W_{n, m}$-models with the $\mathbf{Z}_{2}$-symmetry.

Although the scaling analysis from (7.1) to (7.5) cannot be applied for the case without the $\mathbf{Z}_{2}$-symmetry, we conjecture that in general the $W_{n, m}$-model is equivalent to the $W_{m-1, n+1}$-model by the above identification of the couplings. Notice that this equivalence is based on the interchange of the local coordinates $\lambda$ and $\mu$ in (2.2) (i.e. interchange between $p$ and $p-s$ ). It is also interesting to see how this symmetry is extended for the LG models with multi-poles in the rational potential [Kr2]. In the next section, this equivalence will be checked for the $W_{0,2^{-}}$ and the $W_{1,1}$-models, by calculating the free energy in the small phase space with the different gravity backgrounds given by $\tilde{t}_{N, \beta}=-\delta_{N, 1} \delta_{\beta, \alpha_{0}}$.

We now discuss a formation of singularity in the solution of the dKP hierarchy. As an equation of quasi-linear hyperbolic system, the solution of the dKP hierarchy in general breaks in finite time (formation of shocks). This singularity may represent a phase transition of the matter states due to the gravitational couplings. To regularize this singularity, one needs to include an effect of finite genus, that is, a quantum correction to the phase transition, which can be studied by extending the dKP hierarchy to the Whitham hierarchy [BKo, Du1, Kr2]. The genus in this case would concern with the target space of the TFT, instead of the world sheet. In the following, we illustrate this phase transition (shock formation) for the $W_{1,0}$-model (pure gravity).

In this model, the ring $\mathcal{R}$ consists of only one primary field, that is, the puncture operator $\phi^{0}=\phi_{0}=\mathcal{P}$. Then the string equation (6.8) gives, with $<\sigma_{N-1}\left(\phi^{0}\right) \phi_{0}>=u_{0}^{N} / N !$,

$$
0=\tilde{t}_{0}+\sum_{N \geq 1} \tilde{t}_{N, 0} \frac{u_{0}^{N}}{N !}
$$

where $\tilde{t}_{N, 0}=t_{N, 0}+C_{N, 0}$. For example, if we take $C_{N, 0}=-\delta_{N, N_{0}} N_{0}$ ! with a fixed 
number $N_{0}>0,(7.9)$ expresses the $N_{0}$ th critical phenomena,

$$
u_{0}^{N_{0}}=t_{0}+\sum_{N \geq 1} \frac{u_{0}^{N}}{N !} t_{N, 0}
$$

To discuss a deformation of the critical phenomena based on this equation, let us choose $N_{0}=3$ and set all parameters $t_{N, 0}=0$ except at $N=0$ and 1 , i.e. with $t_{0,0}:=t_{0}$ and $t_{1,0}:=t_{1}$

$$
u_{0}^{3}=t_{0}+u_{0} t_{1}
$$

Fig. 1 shows the bifurcation diagram obtained from (7.11) in the phase space $\left(t_{1}, t_{0}\right)$.

Fig.1 : The bifurcation diagram for the $W_{1,0}$-model with the $N_{0}=3$ criticality.

The curve in the figure gives the points where the derivative $\partial u_{0} / \partial t_{0}$ becomes infinity. This indicates that the criticality at $t_{0}=0$ can be resolved by taking the gravitational coupling to be $t_{1}<0$, and when $t_{1}>0$ it bifurcates into two regions bounded by the singular curve. The region including the $t_{0}$-axis corresponds to the regular state of the matter field, while the region including the positive $t_{1}$-axis 
gives a different state which may imply a genus creation in the target space. The free energy of this system is then caluculated as,

$$
F=\frac{4}{105} t_{0} t_{1}^{2}+\left(\frac{9}{28} t_{0}^{2}+\frac{4}{105} t_{1}^{3}\right) u_{0}+\frac{27}{140} t_{0} t_{1} u_{0}^{2}
$$

where $u_{0}$ is given by the solution of (7.11). In a future communication, we will discuss a process of this bifurcation.

\section{$\S 8$. Examples}

In order to demonstrate the results obtained in this paper, we here give the explicit results for several models, which include the $W_{0,1^{-}}, W_{2,0^{-}}$models for the examples with two primaries, and the $W_{0,2^{-}}, W_{1,1^{-}}$models for those with three primaries.

$W_{0,1}$-model :

The $\mathrm{W}$ potential in this case is given by (2.1),

$$
W=p+\frac{v_{-1}}{p-s}
$$

where $v_{-1}$ and $s$ are related to the universal coordinates as (3.6),

$$
v_{-1}=u^{0}=u_{-1}, \quad s=u^{-1}=u_{0} .
$$

Then we have (2.2),

$$
\begin{aligned}
& \lambda=p+\frac{u_{-1}}{p}+\frac{u_{-1} s}{p^{2}}+\cdots, \\
& \mu=\frac{u_{-1}}{p-u_{0}}+u_{0}+\left(p-u_{0}\right) .
\end{aligned}
$$

The flow equation for $t_{-1}$ is then given by (3.10) and (4.31),

$$
\begin{aligned}
\frac{\partial}{\partial t_{-1}}\left(\begin{array}{c}
u_{-1} \\
u_{0}
\end{array}\right) & =\left(\begin{array}{cc}
0 & 1 \\
1 / u_{-1} & 0
\end{array}\right) \frac{\partial}{\partial t_{0}}\left(\begin{array}{c}
u_{-1} \\
u_{0}
\end{array}\right) \\
& =\frac{\partial}{\partial t_{0}}\left(\begin{array}{ll}
0 & 1 \\
1 & 0
\end{array}\right) \nabla H_{0}^{-1},
\end{aligned}
$$


where $\nabla H_{0}^{-1}:=\left(\partial H_{0}^{-1} / \partial u_{-1}, \partial H_{0}^{-1} / \partial u_{0}\right)^{T}$, and the hamiltonian function $H_{0}^{-1}$ is given by $(4.16 \mathrm{~b})$,

$$
H_{0}^{-1}=<\phi^{-1}>_{0}:=\frac{\partial F_{0}}{\partial u_{-1}}=\frac{1}{2} u_{0}^{2}+u_{-1}\left(\log u_{-1}-1\right)
$$

Here the universal free energy $F_{0}$ can be found as follows: With the hamiltonian $H_{0}^{0}=<\phi^{0}>_{0}=\partial F_{0} / \partial u_{0}=u_{0} u_{-1}$ for the identity flow, we obtain

$$
F_{0}=\frac{1}{2} u_{0}^{2} u_{-1}+\frac{1}{2} u_{-1}^{2}\left(\log u_{-1}-\frac{3}{2}\right) .
$$

The hamiltonian for the descendant field $H_{1}^{-1}$ of (5.11) becomes

$$
H_{1}^{-1}=<\sigma\left(\phi^{-1}\right)>_{0}=\frac{1}{6} u_{0}^{3}+u_{0} u_{-1}\left(\log u_{-1}-1\right)
$$

The 2-point functions $<\phi^{i} \phi^{j}>$ then become

$$
\begin{aligned}
& <\phi^{0} \phi^{0}>=u_{-1}, \quad<\phi^{0} \phi^{-1}>=u_{0}, \quad<\phi^{-1} \phi^{-1}>=\log u_{-1}, \\
& <\sigma_{1}\left(\phi^{0}\right) \phi^{0}>=u_{0} u_{-1}, \quad<\sigma_{1}\left(\phi^{0}\right) \phi^{-1}>=u_{-1}+\frac{1}{2} u_{0}^{2}, \\
& <\sigma_{1}\left(\phi^{-1}\right) \phi^{0}>=u_{-1}\left(\log u_{-1}-1\right)+\frac{1}{2} u_{0}^{2}, \quad<\sigma_{1}\left(\phi^{-1}\right) \phi^{-1}>=u_{0} \log u_{-1}, \\
& <\sigma_{1}\left(\phi^{0}\right) \sigma_{1}\left(\phi^{0}\right)>=\frac{1}{2} u_{-1}^{2}+u_{-1} u_{0}^{2}, \\
& <\sigma_{1}\left(\phi^{0}\right) \sigma_{1}\left(\phi^{-1}\right)>=\frac{1}{3} u_{0}^{3}+u_{-1} u_{0} \log u_{-1}, \\
& <\sigma_{1}\left(\phi^{-1}\right) \sigma_{1}\left(\phi^{-1}\right)>=u_{0}^{2} \log u_{-1}+u_{-1}\left\{\left(\log u_{-1}\right)^{2}-2 \log u_{-1}+2\right\}
\end{aligned}
$$

which are put in the string equation (6.8),

$$
\begin{aligned}
& \tilde{t}_{0}+<\phi^{0} \phi_{0}>\tilde{t}_{1,0}+<\phi^{-1} \phi_{0}>\tilde{t}_{1,-1} \\
& \quad+<\sigma_{1}\left(\phi^{0}\right) \phi_{0}>\tilde{t}_{2,0}+<\sigma_{1}\left(\phi^{-1}\right) \phi_{0}>\tilde{t}_{2,-1} \cdots=0 \\
& \tilde{t}_{-1}+<\phi^{0} \phi_{-1}>\tilde{t}_{1,0}+<\phi^{-1} \phi_{-1}>\tilde{t}_{1,-1} \\
& \quad+<\sigma_{1}\left(\phi^{0}\right) \phi_{-1}>\tilde{t}_{2,0}+<\sigma_{1}\left(\phi^{-1}\right) \phi_{-1}>\tilde{t}_{2,-1} \cdots=0 .
\end{aligned}
$$

As we mentioned in Section 2, we can set either $a) t_{0}=t_{\mathcal{P}}$ or $\left.b\right) t_{-1}=t_{\mathcal{P}}$. The case of $a$ ) corresponds to the dKP equation in the form of (8.4), while in the 
case of $\mathrm{b}$ ) the dKP equation is rewritten in the form (3.15) with $\alpha_{0}=-1$,

$$
\begin{aligned}
\frac{\partial}{\partial t_{0}}\left(\begin{array}{c}
\tilde{u}_{-1} \\
\tilde{u}_{0}
\end{array}\right) & =\left(\begin{array}{cc}
0 & e^{\tilde{u}_{0}} \\
1 & 0
\end{array}\right) \frac{\partial}{\partial t_{-1}}\left(\begin{array}{c}
\tilde{u}_{-1} \\
\tilde{u}_{0}
\end{array}\right) \\
& =\frac{\partial}{\partial t_{-1}}\left(\begin{array}{ll}
0 & 1 \\
1 & 0
\end{array}\right) \tilde{\nabla} \tilde{H}_{0}^{0}
\end{aligned}
$$

where we define $\tilde{u}_{\alpha}=<\phi^{\alpha_{0}} \phi_{\alpha}>$ in (4.4) with $\alpha_{0}=-1$,

$$
\tilde{u}_{0}:=<\phi^{-1} \phi_{0}>=\log u_{-1}, \quad \tilde{u}_{-1}:=<\phi^{-1} \phi_{-1}>=u_{0} .
$$

The hamiltonian function $\tilde{H}_{0}^{0}$ in $(8.10)$ is given by

$$
\tilde{H}_{0}^{0}=<\tilde{\phi}^{0}>_{-1}:=\frac{\partial F_{-1}}{\partial \tilde{u}_{0}}=\frac{1}{2} \tilde{u}_{-1}^{2}+e^{\tilde{u}_{0}},
$$

with the free energy $F_{-1}$ in $(4.15 \mathrm{~b})$,

$$
F_{-1}=\frac{1}{2} \tilde{u}_{-1}^{2} \tilde{u}_{0}+e^{\tilde{u}_{0}}
$$

In particular, note the universality of the 2-point function (4.14),

$$
<\phi^{\alpha} \phi^{\beta}>_{0}:=\frac{\partial^{2} F_{0}}{\partial u_{\alpha} \partial u_{\beta}}=\frac{\partial^{2} F_{-1}}{\partial \tilde{u}_{\alpha} \partial \tilde{u}_{\beta}}:=<\tilde{\phi}^{\alpha} \tilde{\phi}^{\beta}>_{-1}
$$

where $\tilde{\phi}^{-1}=1$ and $\tilde{\phi}^{0}=p-u_{0}=e^{\tilde{u}_{0}}\left(p-\tilde{u}_{-1}\right)^{-1}\left(\bmod W^{\prime}\right)$. The string equations corresponding to these cases become as follows:

For the case of a), choosing $C_{N, \alpha}=-\delta_{N, 1} \delta_{\alpha, 0}$ in (6.8),

$$
\begin{aligned}
u_{0} & =t_{\mathcal{P}}+t_{1,0} u_{0}+t_{1,-1} \log u_{-1}+\cdots, \\
u_{-1} & =t_{\mathcal{Q}}+t_{1,0} u_{-1}+t_{1,-1} u_{0}+\cdots
\end{aligned}
$$

where $t_{\mathcal{P}}=t_{-1}$ is the primary coupling of $\mathcal{P}=\phi^{0}=\phi_{-1}$. The free energy (6.14) for the flat solution, say $F\left(t_{\mathcal{P}}, t_{\mathcal{Q}}\right)$, is given by $F_{0}$ in (8.6) with the substitution $u_{0}=t_{\mathcal{P}}, u_{-1}=t_{\mathcal{Q}}$.

For the case of $b$ ), we have with the choice $C_{N, \alpha}=-\delta_{N, 1} \delta_{\alpha,-1}$,

$$
\begin{gathered}
\tilde{u}_{-1}=t_{\mathcal{P}}+t_{1,0} e^{\tilde{u}_{0}}+t_{1,-1} \tilde{u}_{-1} \cdots \\
\tilde{u}_{0}=t_{\mathcal{Q}}+t_{1,0} \tilde{u}_{-1}+t_{1,-1} \tilde{u}_{0} \cdots
\end{gathered}
$$


The free energy for the flat solution is then given by $F_{-1}$ in (8.13) with $\tilde{u}_{-1}=$ $t_{\mathcal{P}}, \quad \tilde{u}_{0}=t_{\mathcal{Q}}$. This is the $\mathrm{CP}^{1}$-model discussed in [DW, EY]. As an example including a gravitational coupling, we calculate the free energy (6.14) for the case with nonzero $t_{1,-1}:=t_{1, \mathcal{P}}[\mathrm{EHY}]$ : From (8.16), we first have

$$
\tilde{u}_{-1}=\frac{t_{\mathcal{P}}}{1-t_{1, \mathcal{P}}}, \quad \tilde{u}_{0}=\frac{t_{\mathcal{Q}}}{1-t_{1, \mathcal{P}}}
$$

Then from (6.14) we obtain the same result as in [EHY],

$$
F\left(t_{\mathcal{P}}, t_{\mathcal{Q}}, t_{1, \mathcal{P}}\right)=\frac{1}{2} \frac{t_{\mathcal{P}}^{2} t_{\mathcal{Q}}}{1-t_{1, \mathcal{P}}}+\left(1-t_{1, \mathcal{P}}\right)^{2} e^{\frac{t_{\mathcal{Q}}}{1-t_{1, \mathcal{P}}}} .
$$

Here the point is that our derivation of the free energy is totally algebraic.

$W_{2,0}$-model :

The $\mathrm{W}$ potential is given by

$$
W=\frac{1}{3} p^{3}+v_{1} p+v_{0}
$$

where $v_{0}, v_{1}$ are related to the universal coordinates $u_{0}, u_{1}$ as

$$
v_{0}=u^{1}=u_{0}, \quad v_{1}=u^{0}=u_{1} .
$$

The flow equation for $t_{1}$ is given by

$$
\frac{\partial}{\partial t_{1}}\left(\begin{array}{l}
u_{1} \\
u_{0}
\end{array}\right)=\left(\begin{array}{cc}
0 & 1 \\
-u_{1} & 0
\end{array}\right) \frac{\partial}{\partial t_{0}}\left(\begin{array}{l}
u_{1} \\
u_{0}
\end{array}\right) .
$$

In this model, we obtain the following two flat solutions and the corresponding free energies:

a) With $u_{\alpha}=<\phi^{0} \phi_{\alpha}>$ for $\alpha=0$ and 1 ,

$$
F_{0}=\frac{1}{2} u_{1} u_{0}^{2}-\frac{1}{24} u_{1}^{4}
$$

b) With $\tilde{u}_{0}:=<\phi^{1} \phi_{0}>=-u_{1}^{2} / 2, \tilde{u}_{1}:=<\phi^{1} \phi_{1}>=u_{0}$,

$$
F_{1}=\frac{1}{2} \tilde{u}_{0} \tilde{u}_{1}^{2}+\frac{1}{15}\left(-2 \tilde{u}_{0}\right)^{5 / 2}
$$

Note in this case that $F_{1}$ includes an algebraic singularity which indicates a critical phenomena. 
$W_{0,2}$-model:

The $\mathrm{W}$ potential is given by

$$
W=p+\frac{v_{-1}}{p-s}+\frac{v_{-2}}{2(p-s)^{2}}
$$

with the relation,

$$
v_{-1}=u^{0}=u_{-1}, \quad\left(v_{-2}\right)^{1 / 2}=u^{-2}=u_{-2}, \quad s=u^{-1}=u_{0}
$$

The flow equations in the hierarchy are

$$
\begin{aligned}
\frac{\partial}{\partial t_{-1}}\left(\begin{array}{c}
u_{-1} \\
u_{0} \\
u_{-2}
\end{array}\right) & =\left(\begin{array}{ccc}
0 & 1 & 0 \\
0 & 0 & 1 / u_{-2} \\
1 / u_{-2} & 0 & -u_{-1} / u_{-2}^{2}
\end{array}\right) \frac{\partial}{\partial t_{0}}\left(\begin{array}{c}
u_{-1} \\
u_{0} \\
u_{-2}
\end{array}\right) \\
& =\frac{\partial}{\partial t_{0}}\left(\begin{array}{ccc}
0 & 1 & 0 \\
1 & 0 & 0 \\
0 & 0 & 1
\end{array}\right) \nabla H_{0}^{-1}, \\
\frac{\partial}{\partial t_{-2}}\left(\begin{array}{c}
u_{-1} \\
u_{0} \\
u_{-2}
\end{array}\right) & =\left(\begin{array}{ccc}
0 & 0 & 1 \\
1 / u_{-2} & 0 & -u_{-1} / u_{-2}^{2} \\
-u_{-1} / u_{-2}^{2} & 1 & u_{-1}^{2} / u_{-2}^{3}
\end{array}\right) \frac{\partial}{\partial t_{0}}\left(\begin{array}{c}
u_{-1} \\
u_{0} \\
u_{-2}
\end{array}\right) \\
& =\frac{\partial}{\partial t_{0}}\left(\begin{array}{ccc}
0 & 1 & 0 \\
1 & 0 & 0 \\
0 & 0 & 1
\end{array}\right) \nabla H_{0}^{-2},
\end{aligned}
$$

where the hamiltonian functions are given by

$$
\begin{aligned}
& H_{0}^{-1}=<\phi^{-1}>_{0}=\frac{\partial F_{0}}{\partial u_{-1}}=\frac{1}{2} u_{0}^{2}+u_{-1} \log u_{-2}, \\
& H_{0}^{-2}=<\phi^{-2}>_{0}=\frac{\partial F_{0}}{\partial u_{-2}}=u_{0} u_{-2}+\frac{1}{2} \frac{u_{-1}^{2}}{u_{-2}} .
\end{aligned}
$$

Here the free energy $F_{0}$ is given by

$$
F_{0}=\frac{1}{2} u_{0}^{2} u_{-1}+\frac{1}{2} u_{-2}^{2} u_{0}+\frac{1}{2} u_{-1}^{2} \log u_{-2} .
$$

In this model, there are three flat solutions:

a) With the choice $C_{N, \alpha}=-\delta_{N, 1} \delta_{\alpha, 0}$, we obtain the universal free energy $F_{0}$ given by (8.28). The free energy $F\left(t_{\alpha}: \alpha=0,-1,-2\right)$ in $(6.14)$ is then given by $F_{0}$ with $u_{\alpha}=t_{\alpha}$, in particular, $t_{0}=t_{\mathcal{P}}$. 
b) With $C_{N, \alpha}=-\delta_{N, 1} \delta_{\alpha,-1}$,

$$
F_{-1}=\frac{1}{2} \tilde{u}_{-1} \tilde{u}_{-2}^{2}+\frac{1}{2} \tilde{u}_{0} \tilde{u}_{-1}^{2}-\frac{1}{24} \tilde{u}_{-2}^{4}+\tilde{u}_{-2} e^{\tilde{u}_{0}},
$$

where the new variables $\tilde{u}_{\alpha}, \alpha=0,-1,-2$ are defined by

$$
\begin{aligned}
\tilde{u}_{-1} & :=<\phi^{-1} \phi_{-1}>=u_{0}, \\
\tilde{u}_{0} & :=<\phi^{-1} \phi_{0}>=\log u_{-2}, \\
\tilde{u}_{-2} & :=<\phi^{-1} \phi_{-2}>=u_{-1} / u_{-2} .
\end{aligned}
$$

Note here that $<\phi^{\alpha} \phi^{\beta}>=<\tilde{\phi}^{\alpha} \tilde{\phi}^{\beta}>_{-1}=\partial^{2} F_{-1} / \partial \tilde{u}_{\alpha} \partial \tilde{u}_{\beta}$, and the free energy is given by $F\left(t_{\alpha}\right)=F_{-1}\left(\tilde{u}_{\alpha}=t_{\alpha}\right)$ with $t_{-1}=t_{\mathcal{P}}$.

c) With $C_{N, \alpha}=-\delta_{N, 1} \delta_{\alpha,-2}$,

$$
F_{-2}=\frac{1}{6} \tilde{u}_{-1} \tilde{u}_{0}^{3}+\frac{1}{6} \tilde{u}_{-2}^{3}+\tilde{u}_{0} \tilde{u}_{-1} \tilde{u}_{-2}+\frac{1}{2} \tilde{u}_{-1}^{2}\left(\log \tilde{u}_{-1}-\frac{3}{2}\right),
$$

where the new variables are

$$
\begin{aligned}
\tilde{u}_{-1} & :=<\phi^{-2} \phi_{-1}>=u_{-2}, \\
\tilde{u}_{0} & :=<\phi^{-2} \phi_{0}>=u_{-1} / u_{-2}, \\
\tilde{u}_{-2} & :=<\phi^{-2} \phi_{-2}>=u_{0}-\frac{1}{2}\left(u_{-1} / u_{-2}\right)^{2} .
\end{aligned}
$$

The free energy is given in the same way as before, and $t_{-2}=t_{\mathcal{P}}$.

$W_{1,1}$-model :

The $\mathrm{W}$ potential is

$$
W=\frac{p^{2}}{2}+v_{0}+\frac{v_{-1}}{p-s},
$$

where

$$
v_{0}=u^{0}=u_{0}, \quad v_{-1}=u^{1}=u_{-1}, \quad s=u^{-1}=u_{1} .
$$

This model has been recently studied in [Du2, KO]. Here we show that the flat solutions of this model coincide with those of the $W_{0,2}$-model just discussed (the equivalent pair discussed in Section 7). With (8.33), the flow equations (3.10) are

$$
\begin{aligned}
\frac{\partial}{\partial t_{1}}\left(\begin{array}{c}
u_{-1} \\
u_{0} \\
u_{1}
\end{array}\right) & =\left(\begin{array}{ccc}
u_{1} & 0 & u_{-1} \\
1 & 0 & 0 \\
0 & 1 & u_{1}
\end{array}\right) \frac{\partial}{\partial t_{0}}\left(\begin{array}{c}
u_{-1} \\
u_{0} \\
u_{1}
\end{array}\right), \\
\frac{\partial}{\partial t_{-1}}\left(\begin{array}{c}
u_{-1} \\
u_{0} \\
u_{1}
\end{array}\right) & =\left(\begin{array}{ccc}
0 & 1 & u_{1} \\
0 & 0 & 1 \\
1 / u_{-1} & 0 & 0
\end{array}\right) \frac{\partial}{\partial t_{0}}\left(\begin{array}{c}
u_{-1} \\
u_{0} \\
u_{1}
\end{array}\right) .
\end{aligned}
$$


The three flat solutions are as follows:

a) With $C_{N, \alpha}=-\delta_{N, 1} \delta_{\alpha, 0}$,

$$
F_{0}=\frac{1}{6} u_{-1} u_{1}^{3}+\frac{1}{6} u_{0}^{3}+u_{1} u_{0} u_{-1}+\frac{1}{2} u_{-1}^{2}\left(\log u_{-1}-\frac{3}{2}\right)
$$

This gives the same free energy $F\left(t_{\mathcal{P}}, t_{\mathcal{Q}_{1}}, t_{\mathcal{Q}_{2}}\right)$ as that in the case c) of the $W_{0,2^{-}}$ model, if we identify the variables $u_{\alpha}$ in this model with $\tilde{u}_{\beta}, \beta=-(\alpha+2)(\bmod 3)$, in the $W_{0,2}$-model as

$$
t_{\mathcal{P}}:=u_{0}=\tilde{u}_{-2}, \quad t_{\mathcal{Q}_{1}}:=u_{1}=\tilde{u}_{0}, \quad t_{\mathcal{Q}_{2}}:=u_{-1}=\tilde{u}_{-1} .
$$

b) With $C_{N, \alpha}=-\delta_{N, 1} \delta_{\alpha,-1}$,

$$
F_{-1}=\frac{1}{2} \tilde{u}_{-1} \tilde{u}_{0}^{2}+\frac{1}{2} \tilde{u}_{1} \tilde{u}_{-1}^{2}-\frac{1}{24} \tilde{u}_{0}^{4}+\tilde{u}_{0} e^{\tilde{u}_{1}},
$$

where

$$
\begin{aligned}
\tilde{u}_{-1} & :=<\phi^{-1} \phi_{-1}>=u_{0}+u_{1}^{2} / 2, \\
\tilde{u}_{0} & :=<\phi^{-1} \phi_{0}>=u_{1}, \\
\tilde{u}_{1} & :=<\phi^{-1} \phi_{1}>=\log u_{-1} .
\end{aligned}
$$

The free energy is the same as in the case b) of the $W_{0,2}$-model.

c) With $C_{N, \alpha}=-\delta_{N, 1} \delta_{\alpha, 1}$,

$$
F_{1}=\frac{1}{2} \tilde{u}_{-1} \tilde{u}_{1}^{2}+\frac{1}{2} \tilde{u}_{1} \tilde{u}_{0}^{2}+\frac{1}{2} \tilde{u}_{-1}^{2} \log \tilde{u}_{0}
$$

where

$$
\begin{aligned}
\tilde{u}_{-1} & :=<\phi^{1} \phi_{-1}>=u_{1} u_{-1}, \\
\tilde{u}_{0} & :=<\phi^{1} \phi_{0}>=u_{-1}, \\
\tilde{u}_{1} & :=<\phi^{1} \phi_{1}>=u_{0}+u_{1}^{2} / 2 .
\end{aligned}
$$

The free energy is again the same as in the case a) of the $W_{0,2}$-model. 


\section{Appendix A: Calculus including log-terms}

Here we give the explicit calculations of quantities including log-terms, for example, the generators $Q_{N}^{-1}$ in (5.7), and the 1-point function (4.16b) of the corresponding fields $\sigma_{N}\left(\phi^{-1}\right)$.

We first define the projection symbols $[\cdot]_{+}$and $[\cdot]_{-}$in (2.5) in terms of the contour integrals. Let $f(p) \in \mathbf{C}\left[p,(p-s)^{-1}\right]$, that is, $f(p)$ is a rational function given by

$$
f(p)=\sum_{k \geq 0} a_{k} p^{k}+\sum_{l \geq 1} \frac{b_{l}}{(p-s)^{l}} .
$$

Then the + and - projections are defined by

$$
\begin{aligned}
& {[f(p)]_{+}:=\sum_{k \geq 0} a_{k} p^{k},} \\
& {[f(p)]_{-}:=\sum_{l \geq 1} \frac{b_{l}}{(p-s)^{l}} .}
\end{aligned}
$$

The coefficients $a_{l}$ and $b_{l}$ are obtained by

$$
\begin{aligned}
a_{k} & =\underset{p=\infty}{\operatorname{res}}\left[\frac{f(p)}{p^{k+1}}\right]:=\frac{1}{2 \pi i} \oint_{C_{\infty}} \frac{f(p)}{p^{k+1}} d p, \\
b_{l} & =\underset{p=s}{\operatorname{ees}}\left[f(p)(p-s)^{l-1}\right]:=\frac{1}{2 \pi i} \oint_{C_{s}} f(p)(p-s)^{l-1} d p,
\end{aligned}
$$

where $C_{\infty}$ and $C_{s}$ are the contoures oriented in the anti-clockwise about $p=\infty$ and $p=s$, respectively. Then from (A.2) and (A.3), we have

$$
\begin{aligned}
& {[f(p)]_{+}=\frac{1}{2 \pi i} \oint_{C_{\infty}} \frac{f(z)}{z-p} d p} \\
& {[f(p)]_{-}=-\frac{1}{2 \pi i} \oint_{C_{s}} \frac{f(p)}{z-p} d p .}
\end{aligned}
$$

Note that the point $p$ in (A.4) locates between $C_{\infty}$ and $C_{s}$. Eqs.(A.4) give explicit formulae for the projections of the rational functions in $\mathbf{C}\left[p,(p-s)^{-1}\right]$.

Now let us consider the case including $\log$-terms, $\log \lambda$ and $\log \mu$. In this case, one has to modify $C_{s}$ in (A.4) to $\tilde{C}_{s}$ which is taken to surround a branch 
cut between $p=s$ and $p=\infty$. The main idea of defining these terms is to regularize the log-terms by adding and subtracting the singular parts of these terms. For the formula $Q_{N}^{-1}$ in (5.7) is then calculated as follows: We first write $\log \lambda=\log (\lambda / p)+\log p$, and $\log \mu=\log [\mu(p-s)]-\log (p-s)$. Then we have

$$
\begin{aligned}
Q_{N}^{-1}= & {\left[\frac{W^{N}}{N !}\left(\log \lambda-c_{N}\right)\right]_{+}-\left[\frac{W^{N}}{N !}\left(\log \mu-d_{N}\right)\right]_{-} } \\
= & {\left[\frac{W^{N}}{N !}\left(\log \frac{\lambda}{p}-c_{N}\right)\right]_{+}-\left[\frac{W^{N}}{N !}\left(\log [\mu(p-s)]-d_{N}\right)\right]_{-} } \\
& +\left[\frac{W^{N}}{N !} \log p\right]_{+}+\left[\frac{W^{N}}{N !} \log (p-s)\right]_{-}
\end{aligned}
$$

The first two terms are well defined and give polynomials in $\mathbf{C}\left[p,(p-s)^{-1}\right]$, while the last two terms may be computed by deforming the contour in the integrals (A.4), i.e. with $C_{s} \rightarrow \tilde{C}_{s}$

$$
\begin{aligned}
& {\left[\frac{W^{N}}{N !} \log p\right]_{+}+\left[\frac{W^{N}}{N !} \log (p-s)\right]_{-}} \\
& :=\frac{1}{2 \pi i N !} \oint_{C_{\infty}} \frac{W(z)^{N} \log z}{z-p} d z-\frac{1}{2 \pi i N !} \oint_{\tilde{C}_{s}} \frac{W(z)^{N} \log (z-s)}{z-p} d z \\
& =\frac{1}{2 \pi i N !} \oint_{C_{\infty}} \frac{W(z)^{N}}{z-p} \log \left(\frac{z}{z-s}\right) d z+\frac{W(p)^{N}}{N !} \log (p-s) .
\end{aligned}
$$

Thus $Q_{N}^{-1}$ includes the log-term, and is calculated as

$$
\begin{aligned}
Q_{N}^{-1}= & {\left[\frac{W^{N}}{N !}\left(\log \frac{\lambda}{p-s}-c_{N}\right)\right]_{+}-\left[\frac{W^{N}}{N !}\left(\log [\mu(p-s)]-d_{N}\right)\right]_{-} } \\
& +\frac{W(p)^{N}}{N !} \log (p-s) .
\end{aligned}
$$

In particular, we see from (A.7) that $Q^{-1}=Q_{0}^{-1}=\log (p-s)$ in (2.5). Note here that the fields $\sigma_{N}\left(\phi^{-1}\right)=\partial Q_{N}^{-1} / \partial p$ are all well-defined as elements in the ring $(2.10), \mathcal{R}=\mathbf{C}\left[p,(p-s)^{-1}\right] / W^{\prime}(p)$. 
In a similar way as above, the residue formula including the log-terms can be also explicitly expressed by the contour integrals. Let $f(p) \in \mathbf{C}\left[p,(p-s)^{-1}\right]$. We then want to give a precise meaning of the quantity, e.g. (5.11),

$$
\underset{p=\infty}{\operatorname{res}}[f(p) \log \lambda]+\underset{p=s}{\operatorname{res}}[f(p) \log \mu] .
$$

By the regularizations for $\log \lambda$ and $\log \mu$, we obtain

$$
\begin{aligned}
& \underset{p=\infty}{r e s}\left[f(p) \log \frac{\lambda}{p}\right]+\underset{p=s}{r e s}[f(p) \log [\mu(p-s)]]+\frac{1}{2 \pi i} \oint_{C_{\infty}} f(p)[\log p-\log (p-s)] d p \\
& =\underset{p=\infty}{\operatorname{ees}}\left[f(p) \log \frac{\lambda}{p-s}\right]+\underset{p=s}{\operatorname{res}}[f(p) \log \{\mu(p-s)\}] .
\end{aligned}
$$

Note that the contour integral in (A.9) gives $\int_{0}^{s}[f(p)]_{+} d p$ which was previously obtained in $[\mathrm{AK}]$, i.e.

$$
\oint_{C_{\infty}} f(p)[\log p-\log (p-s)] d p=\int_{0}^{s} d z \oint_{C_{\infty}} \frac{f(p)}{p-z} d p .
$$

\section{Appendix B: The dKP hierarchy}

In this Appendix, we give a brief summary of the dKP theory as a quasiclassical limit of the KP theory. For a simplicity, we consider here the original form of the KP hierarchy, and not a constrained hierarchy discussed recently in [BX, AFNV, OS], which is directly related to our dKP hierarchy in the dispersionless limit. The KP theory without constraint may be formulated as follows:

Let $L$ be a formal pseudo-differential operator given by

$$
L=\partial+\sum_{i=0}^{\infty} A^{i} \partial^{-(i+1)},
$$

where $A^{i}=A^{i}\left(X, T_{1}, T_{2}, \cdots\right)$, the symmbol $\partial$ implies the derivative with respect to $X$, and $\partial^{-1} \partial=\partial \partial^{-1}=1$. The operation with $\partial^{i}$ is given by a generalized Leibnitz rule,

$$
\partial^{i} F=\sum_{j=0}^{\infty}\left(\begin{array}{c}
i \\
j
\end{array}\right) \frac{\partial^{j} F}{\partial X^{j}} \partial^{i-j} \quad, \quad i \in \mathbf{Z}
$$


The KP hierarchy is then defined by the so-called Lax formula,

$$
\frac{\partial L}{\partial T_{n}}=\left[B_{n}, L\right]:=B_{n} L-L B_{n}, \quad n=0,1, \cdots
$$

where the differential operator $B_{n}$ is given by the differential part of $L^{n+1} /(n+1)$, denoted by

$$
B_{n}=\frac{1}{n+1}\left[L^{n+1}\right]_{+}
$$

The hierarchy (B.3) is also given by the compatibility conditions of the following linear equations for the wave function $\Psi\left(T_{0}, T_{1}, \cdots\right)$ with $T_{0}=X$, and $\frac{\partial \lambda}{\partial T_{n}}=0$;

$$
\begin{gathered}
L \Psi=\lambda \Psi \\
\frac{\partial \Psi}{\partial T_{n}}=B_{n} \Psi
\end{gathered}
$$

Note that (B.5b) gives an iso-spectral deformation of the operator $L$ in (B.5a). Then, the dKP hierarchy can be obtained from the "quasi-classical" limit of the KP theory as follows [Ko2]: Let $\hbar$ be a small parameter (the Plank constant), and introduce the variables,

$$
\begin{aligned}
& t_{n}:=\hbar T_{n}, \quad \text { for } \quad n=0,1, \cdots, \\
& a^{i}=a^{i}\left(t_{0}, t_{1}, \cdots\right):=A^{i}\left(T_{0}, T_{1}, \cdots\right), \quad \text { for } \quad i=0,1, \cdots,
\end{aligned}
$$

which lead to the replacement $\frac{\partial}{\partial T_{n}}=\hbar \frac{\partial}{\partial t_{n}}$. Then write the wave function $\Psi$ in (B.5) to be the WKB form,

$$
\Psi\left(T_{0}, T_{1}, \cdots\right)=\exp \left\{\frac{1}{\hbar} S\left(t_{0}, t_{1}, \cdots\right)\right\} .
$$

where the function $\mathrm{S}$ is called the action, and plays a fundamental role of the dKP theory. With (B.7) the quasi-classical limit leads to, for $i \in \mathbf{Z}$,

$$
\frac{\partial_{X}{ }^{i} \Psi}{\Psi}=\hbar^{i} \frac{\partial_{x}{ }^{i} \Psi}{\Psi} \rightarrow p^{i}, \quad \text { as } \quad \hbar \rightarrow 0 .
$$

where $p$ is the momentum function defined by

$$
p=\frac{\partial S}{\partial x}
$$


From (B.8), eqs.(B.5) in the limit become

$$
\begin{aligned}
& \lambda=p+\frac{a^{0}}{p}+\frac{a^{1}}{p^{2}}+\cdots, \\
& \frac{\partial p}{\partial t_{n}}=\frac{\partial Q^{n}}{\partial x}
\end{aligned}
$$

where $Q^{n}$ given by $\lim _{\hbar \rightarrow \infty}\left[B_{n} \Psi / \Psi\right]$ is the polynomial part of $\lambda^{n+1} /(n+1)$ in $p$, and we denote $Q^{n}=\left[\lambda^{n+1} /(n+1)\right]_{+}$as in (2.5). Note that (B.10) is the HamiltonJacobi equation for the wave equation (B.5), and it defines the dKP hierarchy. In this formulation, $\lambda$ is considered to be a constant given by the spectral parameter of $L$. Note that the formulation we used in the text is different from the one given here, that is, the momentum function $p$ in the text is considered as a parameter instead of $\lambda$. However, these formulations are of course equivalent, and indeed they are connected as a cannonical change of variables: Namely consider the differential three-forms ( $\infty$-forms in general),

$$
d \lambda \wedge d p \wedge d x=d Q^{n} \wedge d \lambda \wedge d t_{n}
$$

which leads to both (B.10) and the dKP hierarchy in the form (2.4) by considering the independent variables to be either $\left(\lambda, t_{n}, x\right)$ or $\left(p, t_{n}, x\right)$, and comparing the coefficients of $d \lambda \wedge d t_{n} \wedge d x$ or $d p \wedge d t_{n} \wedge d x$. Now it is clear from (B.10) that the function $p$ gives the conserved densities of the hierarchy (Theorem 3.1). Also the compatibility conditions among the flows in (B.10), which are now given by

$$
\frac{\partial Q^{i}}{\partial t_{j}}=\frac{\partial Q^{j}}{\partial t_{i}} .
$$

Note from (B.9) and (B.10b) that $Q^{i}$ is written in the form with the action $S$,

$$
Q^{i}=\frac{\partial S}{\partial t_{i}}, \quad \text { for } \quad n=0,1, \cdots
$$

Writing $Q^{i}$ in a Laurent series of $\lambda$, we have

$$
Q^{i}=\frac{1}{i+1}\left[\lambda^{i+1}\right]_{+}:=\frac{1}{i+1} \lambda^{i+1}-\sum_{j=0}^{\infty} \frac{1}{\lambda^{j+1}} G^{i j} .
$$

Here the coefficients $G^{i j}$ can be calculated by the residue form,

$$
G^{i j}=-\underset{\lambda=\infty}{\operatorname{res}}\left[Q^{i} \lambda^{j}\right]=\frac{1}{j+1} \underset{p=\infty}{\operatorname{res}}\left[\lambda^{i+1} \frac{\partial Q^{i}}{\partial p}\right],
$$


which is just (3.11a), and also shows $G^{i j}=G^{j i}$. We then see that the action $S$ can be written in terms of the free energy $F$,

$$
S=\sum_{i=0}^{\infty} t_{i} \frac{\lambda^{i+1}}{i+1}-\sum_{j=0}^{\infty} \frac{1}{\lambda^{j+1}} \frac{\partial F}{\partial t_{j}}
$$

Here the free energy is defined by (4.2), $G^{i j}=\partial^{2} F / \partial t_{i} \partial t_{j}$. The existence of the free energy is a consequence of the integrability (B.12), i.e. (3.14),

$$
\frac{\partial G^{i j}}{\partial t_{k}}=\frac{\partial G^{k j}}{\partial t_{i}}
$$

Noticing the scale invariance of (B.17) under $t_{i} \rightarrow \epsilon t_{i}$, we see that the functions $G^{i j}$ are homogeneous functions of degree zero, and so that the free energy $F$ is of degree two , i.e. (6.16) [Kr1, TT2]. This implies

$$
\sum_{i=0}^{\infty} t_{i} \frac{\partial}{\partial t_{i}} F=2 F
$$

Then taking the derivative of (B.18) with respect to $t_{j}$ leads to

$$
\sum_{i=0}^{\infty} t_{i} \frac{\partial^{2}}{\partial t_{i} \partial t_{j}} F=\frac{\partial F}{\partial t_{j}}
$$

and, using (B.18) once again, we obtain the formula (6.14) of the free energy, i.e.

$$
F=\frac{1}{2} \sum_{i, j=0}^{\infty} t_{i} t_{j} G^{i j}
$$

Note also that using the formula (B.15) the free energy $F$ can be written in the form $[\mathrm{Kr} 1]$,

$$
F=\frac{1}{2} \underset{p=\infty}{\operatorname{res}}\left[S^{+} \frac{\partial S_{+}}{\partial p}\right],
$$

where $S^{+}:=\sum_{i=0}^{\infty} t_{i} \lambda^{i+1} /(i+1)$, and $S_{+}=\sum_{i=0}^{\infty} t_{i} Q^{i}$. 


\section{References}

[AK] S. Aoyama, and Y. Kodama: Mod. Phys. Lett. A9 (1994) 2481.

[ANPV] H. Aratyn, E. Nissimov, S. Pacheva, and I. Varysburg: Phys. Lett. B294 (1992)167.

[BDKS] E. Brezin, M. Douglas, V. Kazakov, and S. Shenker: Phys. Lett. B237 (1990) 43.

[BDSS] T. Banks, M. Douglas, N. Seiberg and S. Shenker, Phys. Lett. B238 (1990) 279.

[BKa] E. Brezin, and V. Kazakov: Phys. Lett. B236 (1990) 144.

[BKo] A. M. Block, and Y. Kodama: SIAM J. Appl. Math. 52 (1992) 909.

[BX] L. Bonora, and C. S. Xiong: Int. J. Mod. Phys. A8 (1993) 2973; Nucl. Phys. B405 (1993) 191; Phys. Lett. B317 (1993) 329.

[CGM] C. Crnkovic, P. Ginsparg and G. Moore, Phys. Lett. B237 (1990) 196.

[Da] F. David, Mod. Phys. Lett. A3 (1988) 1651.

[Do] M. Douglas: Phys. Lett. B238 (1990) 176.

[Du1] B. Dubrovin: Comm. Math. Phys. 145(1992)195; Nucl. Phys. B379 (1992) 627.

[Du2] B. Dubrovin: Geometry of 2D topological field theory, SISSA-84/94/FM, hep-th/9407018.

[DK] J. Distler and H. Kawai, Nucl. Phys. B321 (1989) 509.

[DS] M. Douglas, and S. Shenker: Nucl. Phys. B335 (1990) 635.

[DVV1] R. Dijkgraaf, H. Verlinde and E. Verlinde: Nucl. Phys. B352 (1991) 59.

[DVV2] R. Dijkgraaf, H. Verlinde and E. Verlinde: Nucl. Phys. B348 (1991) 435.

[DW] R. Dijkgraaf and E. Witten: Nucl. Phys. B342 (1990) 486.

[EHY] T. Eguchi, K. Hori, and S. -K. Yang: Topological $\sigma$-model and large-N matrix model, UT-700, hep-th/9503017.

[EKYY] T. Eguchi, H. Kanno, Y. Yamada and S.-K. Yang: Phys. Lett. B305 (1993) 235.

[EY] T. Eguchi, and S.-K. Yang: Mod. Phys. Lett. A9 (1994) 2893.

[EYY1] T. Eguchi, Y. Yamada and S.-K. Yang: Mod. Phys. Lett. A8 (1993) 1627.

[EYY2] T. Eguchi, Y. Yamada and S.-K. Yang: On the Genus Expansion in the Topological String Theory, UTHEP-275, hep-th/9405106. 
[FGZ] P. Di Francesco, P. Ginsparg, and J. Zinn-Justin: 2D Gravity and Random Matrices, LA-UR-93-1722, SPhT/93-061, hep-th/9306153.

[FK] P. D. Francesco, and D. Kutasov, Nucl. Phys. B342 (1990) 589.

[FKN] M. Fukuma, H. Kawai, and R. Nakayama: Int. J. Mod. Phys. A6 (1991) 1385.

[GGPZ] P. Ginsparg, M. Goulian, M. R. Plesser, and J. Zinn-Justin: Nucl. Phys. B342 (1990) 539.

[GM] D. Gross, and A. Migdal: Nucl. Phys. B340 (1990) 333.

[Ka] V. Kazakov: Mod. Phys. Lett. A4 (1989) 2125.

[Ko1] Y. Kodama: Phys. Lett. A147 (1990) 477.

[Ko2] Y. Kodama: Prog. Theor. Phys. Supp. 94 (1988) 184; Phys Lett. A129 (1988) 223.

[Kr1] I. M. Krichever: Comm. Math. Phys. (1991) 415.

[Kr2] I. M. Krichever: Comm. Pure. Appl. Math. 47 (1994) 437.

[KG] Y. Kodama and J. Gibbons: Phys. Lett. A135 (1989) 167; Integrability of the dispersionless KP hierarchy, Proceedings of the workshop "Non-linear Processes in Physics" (World Scientific, 1990) 166.

[KO] H. Kanno, and Y. Ohta: Topological strings with scaling violation and Toda lattice hierarchy, hep-th/9502029.

[KPZ] V.G. Knizhnik, A.M. Polyakov and A.B. Zamolodchikov, Mod. Phys. Lett. A3(1988)819.

[Lo] A. Losev: Theor. Math. Phys. 95 (1993) 595.

[LP] A. Losev and L. Polyubin: On connection between topological LandauGinzburg gravity and integrable systems, hep-th/9305079.

[OS] W. Oevel, and W. Strampp: Comm. Math. Phys. 157 (1993) 51.

[S] I. A. B. Strachan: The Moyal bracket and the dispersionless limit of the KP hierarchy, hep-th/9410048.

[SV] M. V. Saveliev, and A. M. Vershik: Comm. Math. Phys. 126 (1989) 367.

[T] K. Takasaki: Lett. Math. Phys. 29 (1993) 111.

[TT1] K. Takasaki and T. Takebe: Lett. Math. Phys. 23 (1991) 205.

[TT2] K. Takasaki and T. Takebe: Int. J. Mod. Phys. A. Supp. (1992) 889.

[VW] C. Vafa and N.P. Warner: Phys. Lett. B218 (1989) 51;

[W] E. Witten: Nucl. Phys. B340 (1990) 281. 
[Z] B. Zuber: On Dubrovin topological field theories, SPht 93/147, hep-th 9312209 . 